\title{
Missing links: the functional unification of language and memory (LUM)
}

\author{
Running title: Language and memory unification ( $\mathrm{L} \cup \mathrm{M})^{*}$ \\ Elise Roger ${ }^{\mathrm{a}, *}$, Sonja Banjac ${ }^{\mathrm{a}}$, Michel Thiebaut De Schotten ${ }^{\mathrm{b}, \mathrm{c}}$, Monica Baciu ${ }^{\mathrm{a}}$. \\ a LPNC, Univ. Grenoble Alpes, CNRS UMR 5105, Grenoble, France \\ b Brain Connectivity and Behaviour Laboratory, Sorbonne Universities, Paris, France \\ ${ }^{c}$ Groupe d'Imagerie Neurofonctionnelle, Institut des Maladies Neurodégénératives-UMR 5293, CNRS, CEA \\ University of Bordeaux, Bordeaux, France
}

\begin{abstract}
The field of neurocognition is currently undergoing a significant change of perspective. Traditional neurocognitive models evolved into an integrative and dynamic vision of cognitive functioning. Dynamic integration assumes an interaction between cognitive domains traditionally considered to be distinct. Language and declarative memory are regarded as separate functions supported by different neural systems. However, they also share anatomical structures (notably, the inferior frontal gyrus, the supplementary motor area, the superior and middle temporal gyrus, and the hippocampal complex) and cognitive processes (such as semantic and working memory) that merge to endorse our quintessential daily lives. We propose a new model, "LUM" (i.e., Language/union/Memory), that considers these two functions interactively. We fractionated language and declarative memory into three cognitive dimensions, Embodiment-FormulationInternalization, that communicate reciprocally. We formalized their interactions at the brain level with a connectivity-based approach. This new taxonomy overcomes the modular view of cognitive functioning and reconciles functional specialization with plasticity in neurological disorders.
\end{abstract}

\section{Keywords}

Language, memory, inter-functional relations, interactive model, cognitive neuroscience

${ }^{*}$ Corresponding author: Elise Roger (Ph.D.)

Email address: elise.roger@univ-grenoble-alpes.fr 


\section{Graphical abstract}

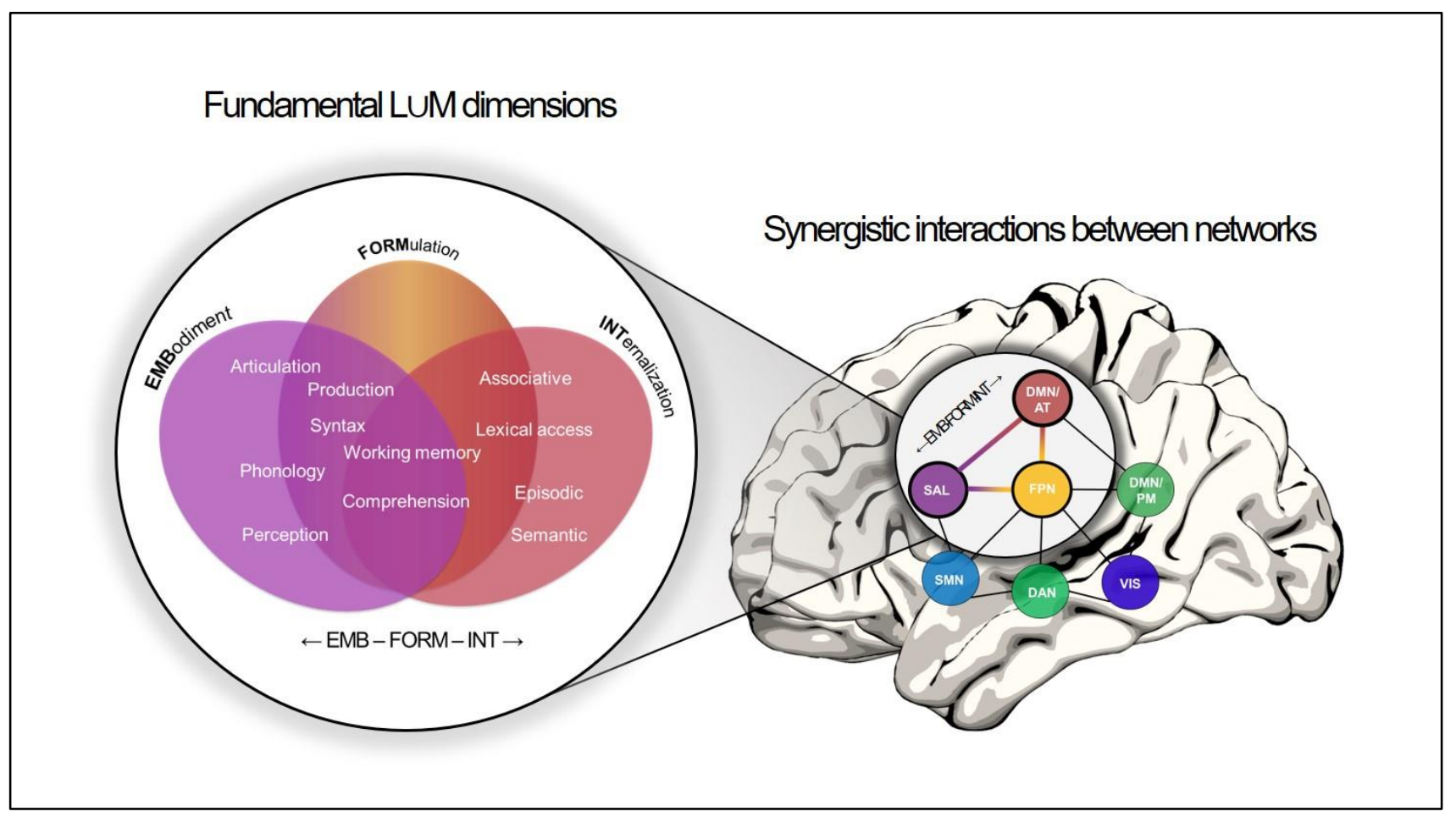

\section{Highlights}

- Language and declarative memory are constantly interacting in a unified and dynamic way

- Their functional interactions are still poorly understood and need to be specified

- We propose a new framework ("LUM") to formalize these missing links

- Interdependence arises from three fundamental dimensions (Embodiment-FormulationInternalization)

- The synergy of specific high-level brain networks underlies the unity between language and declarative memory 


\section{Introduction}

Language and long-term declarative memory (Tulving, 1972) are essential functions of everyday human behavior. The first scientific descriptions date back a little more than a century (e.g., Hermann Ebbinghaus 1885 for memory; Ferdinand de Saussure and his wellknown "Cours de Linguistique générale" 1916 for language) and continue to inspire modern conceptions. Initially, linguists, psychologists, and cognitive scientists investigated language and memory, leading to the usual compartmentalization of these two functions.

Several deep-routed reasons may have contributed to consider these functions as nearly antipodal. Regarding the terminology, language aims at externalization (produce, from Latin producere: lead forward). In contrast, memory subserves internalization (encoding: transcribing inwards). Language is traditionally assumed to involve "domain(s)-specific" processes (specific to language domains, e.g., syntax; Zaccarella \& Friederici, 2015), whereas memory is regarded as a transversal function contingent on "domain(s)-general" processes (Campbell \& Tyler, 2018). The functional epicenters of these two functions based on lesion studies also appear separated anatomically. Language would mainly engage lateral and neocortical cerebral regions (Broca, 1865; Wernicke, 1874), and long-term memory would mainly pertain to mesial and allocortical regions (Milner, 1970; S. Squire, 1984; Figure 1).

Despite this first impression of cognitive and anatomical contrast, several common behavioral phenomena directly emanate from the language-memory overlap. Language skills in children condition their verbal memory (Klemfuss, 2015). Bilingual individuals bind their encoded memories to different languages (Larsen et al., 2002; Marian \& Neisser, 2000). Although a variety of causes can account for verbal paraphasias (e.g., disruption of the earliest stages of lexical processing, such as the conceptual preparation stage; Levelt, 1989; Indefrey, 2012), they can also be explained by a failure of the working memory system, leading to a contamination of production by long-term declarative memory (see Schwering \& MacDonald, 2020 for a review). Episodic false memories and confabulations are verbally generated for the sake of discourse coherence (Conway \& Loveday, 2015). Finally, dissociations between semantic and episodic memory have been reported in patients with semantic dementia (e.g., Graham et al., 2000). However, the frequent scenario of patients with lesions in "language regions" and symptoms in the memory domain or vice versa (e.g., Everts et al., 2010; Jaimes-Bautista et al., 2015) attests to their strong neuropsychological interconnection.

Nevertheless, the neuroscientific community essentially approaches these functions separately. It focuses on psychological concepts or constructs - such as verbal production, comprehension, syntax, semantic, episodic, and working memory - which are conceptually nested (see Box 1). We propose to move towards a more "naturalistic" account of neurocognitive functioning by approaching the cross-functional relationships between language and memory across their fundamental dimensions, neurocognitive operations, and implicated substrates. In contrast to conventional cognition-centered approaches built on theoretical psychological constructs, the "neuro-centric" perspective developed here stands out as an appropriate framework to address the missing links between brain and behavior (Box 2 for an overview of the basic principles that frames our considerations).

How do language and declarative memory interact in a unified way, ensuring our adaptive human behaviors? Owing to the emergence of the brain connectivity study and current connectomic approaches, it is now possible to highlight the neural architecture and interactive pathways underlying these wide-ranging relationships. Here, we chronologically synthesized 
the discovery of language and memory anatomic and functional organization (Sections 2.1 \& 2.2). We then outlined recent elements as a robust foundation for proposing an interactive and unified language and memory functional model (Section 2.3). We present the Language/union/Memory (LUM) model and link it to experimental and theoretical evidence (Section 3). Finally, we illustrate LUM's application in patients and discuss prospects for the future in cognitive and clinical neuroscience (Section 4).

\section{Main legacies of neurocognitive observations}

\subsection{Language and memory coevolution}

Since the beginning of language science, linguists have distinguished two main fields of study: (i) expressive language for syntactic or articulatory aspects of language; and (ii) receptive language for language perception and comprehension. Verbal production (externalization process or "spell-out"; Bolhuis et al., 2014), the expressive language aspect closest to the physical world (de Boer, 2011; Fitch, 2010), is intimately related to embodied or "grounded" cognition (Kempe \& Brooks, 2016). Several past observations have suggested that language production and the emergence of human-specific articulatory gestures may be the direct precursor of syntax (Carstairs-McCarthy, 1999; Studdert-Kennedy, 2005). At the interface between meaning and sound (Ferreira \& Engelhardt, 2006), syntax allows the hierarchical combination of verbal units (e.g., combinations of words, propositions, phrases, sentences) into increasingly complex structures (Friederici et al., 2017). Thus, a complex syntax requires a robust phonological memory system adapted to flexibly organize and maintain the different verbal elements during online processing (Aboitiz et al., 2010).

From an evolutionary perspective, the white matter dorsal pathway composed of superior longitudinal and arcuate fascicles has been particularly extensive in humans compared to nonhuman primates (Goucha et al., 2017; Rilling, 2014). The dorsal circuitry's evolution allowed for the direct control of the vocalization system and made phonological processing more efficient (Aboitiz, 2012). By interconnecting the so-called "Broca and Wernicke's areas", the arcuate fascicle structures the dialogues between abstract structural building (inferior frontal gyrus) and lexical entries or lexically-based argument structures (posterior temporal cortex), promoting the integration of syntactic and semantic information (Goucha et al., 2017). The recruitment and the expansion of such dorsal fascicles could have generated the crucial turning point for exploiting a complex syntactic system and an advanced verbal working memory processing, essential for language acquisition. Humans have extended working memory capacity (Reuland, 2017), including notably inner speech, favoring repetition, and access to consciousness to verbal information (Carruthers, 2013). Thus, working memory can serve as a fundamental unifying function between language production and declarative memory by facilitating conscious introspection. It allows flexible and vivid access to the content of representations in long-term memory (see Brown-Schmidt \& Duff, 2016; Schwering \& MacDonald, 2020) for an in-depth and up-to-date report on verbal working memory).

The emergence of syntax and an adapted working memory system could have led to "open the door" to a broader and richer semantic system. Combined with sophisticated assembling capacities, our semantic system might be the root of the conceptual and propositional organization of complex thought and verbal comprehension (i.e., a receptive system wellsharpened for labeling Boeckx \& Benítez-Burraco, 2014; Hinzen, 2013). For instance, while monkeys can learn to visually categorize images after intensive training (Fabre-Thorpe, 2003), young children are indeed able to learn efficiently new concepts and corresponding words faster 
(Bloom, 2000; Waxman, 2004). This is consistent with the fact that the ventral pathway involved in lexical and semantic processing is less developed in non-human primates (e.g., the inferior longitudinal fascicle joins occipital rather than temporal regions in monkeys; Catani \& Dawson, 2017).

As with the semantic processing, mental time travel, and episodic memory, often referred to as declarative or "declarable" memory (Squire, 2004; Tulving, 1972; Box 1), have co-evolved with language to convey our mental journeys and imaginations (Corballis, 2019). At some point, the system inevitably adapted itself to integrate speech into long-term memory, leading to developing an integrative, unified, and adaptive declarative system in humans. These coevolution mechanisms between expressive-receptive language and memory systems appear to be a critical element of the foundation of human sensus communis (Schaeffer, 1990, for a historical and philosophical description of the theory of knowledge).

\subsection{Contemporary neurofunctional conceptions}

\subsubsection{Language}

Beyond the classic and localizationist, Broca-Wernicke's model and the arcuate fascicle, a vast interconnected network extending outside of the left hemisphere supports the multiple exchanges of information necessary for the production and understanding of language (Figure 1 for the putative role of brain regions and fascicles in language processing). This advanced language representation has challenged the modular and serial views and demonstrated that semantic, phonological, and syntactic processing pertains to parallel large-scale corticosubcortical sub-networks. These specialized systems would be dynamically interconnected (Duffau et al., 2014), allowing rich and flexible language skills.

Recent neurocognitive models of language involve a dual-stream (Duffau et al., 2014; Friederici et al., 2017; Hickok \& Poeppel, 2007; Price, 2012). Temporo-parieto-frontal regions interact along the dorsal pathway through superior longitudinal and arcuate white matter fascicles. As mentioned above, the dorsal stream participates in the conversion of phonological structures into phonetic, articulatory, syntactic, and motor representations and involves verbal working memory. In contrast, occipito-temporo-frontal regions are inter-connected through the uncinate, extreme capsule, inferior longitudinal, and inferior fronto-occipital fascicles along the ventral stream. The ventral stream is mainly involved in the conversion of phonological units into their meaning. Dorsoventral transverse connections have recently been examined further because of their probable involvement in linking the ventral to the dorsal streams (Bullock et al., 2019). The most prominent of them - the middle longitudinal fascicle - is notably highlighted for its double involvement in phonology and semantic processing [e.g., Luo et al., (2020) for evidence in primary progressive aphasia; Hula et al., (2020), in post-stroke aphasia]. The two main streams underlying both production and comprehension are functionally interconnected and tightly coupled (Hickok \& Poeppel, 2007; Silbert et al., 2014) by bundles (or portions of fibers) of the human white-matter backbone (Herbet \& Duffau, 2020) for an extensive hodological model. Figure 1 below provides a comprehensive view of the brain regions and fascicles described in the contemporary language models and their assumed roles.

\subsubsection{Declarative memory}

The observations of brain-injured amnesic patients allowed for the exploration of declarative episodic memory cerebral substrates, with a prominent initial focus on the mesial temporal lobe and the hippocampus (Squire, 1984; Gaffan, 1997 for a critical review). The extensive study of the very well-known Henry Gustav Molaison patient (widely referred to as "H.M." (Scoville \& Milner, 1957) has demonstrated the association between mesial temporal lobe 
lesions and declarative episodic memory deficits (Corkin, 2013; Squire, 2009). More recently, the H.M. case has been revisited according to hodological principles (Fytche \& Catani, 2005). Disconnection analyses revealed damage to various bundles (uncinate, fornix, anterior commissure, and cingulum) and the disconnection of areas not directly affected by the surgery (Thiebaut de Schotten et al., 2015). In line with this observation, a broader functional network involving subcortical but also lateral temporal, (pre)frontal, cingulate, and parietal cortices is known to be involved in declarative memory (Figure 1 for details; see also Danker \& Anderson, 2010; Moscovitch et al., 2006; Sheldon et al., 2016).

The most recent models adopt a "macroscale" neurocognitive view of long-term memory and propose a dual-stream architecture (Duvernoy et al., 2013; Ranganath \& Ritchey, 2012). These models place the medial temporal cortices within a large-scale network (hippocampo-cortical systems) composed of a posterior-medial (PM) and an anterior-temporal (AT) system (PMAT model; Ranganath \& Ritchey, 2012). The posterior medial system includes the retrosplenial cortex involved in the spatial representation of events (Epstein, 2008), posterior cingulate, precuneus, and angular gyrus recruited for the contextual information (Baldassano et al., 2017; Richter et al., 2016; Sreekumar et al., 2018). The cingulum white matter tract mainly connects the posterior medial system. The anterior system includes the perirhinal cortex, the amygdala, and the anterior ventro-temporal and lateral orbitofrontal cortices. The perirhinal cortex supports emotional associations induced by the item (Ritchey et al., 2015). The amygdala binds item features with emotion (Yonelinas \& Ritchey, 2015). The anterior ventro-temporal and lateral orbitofrontal cortices are engaged in object representation and assess items' affective significance and memory decision-making (Libby et al., 2014). The uncinate, the inferior longitudinal fascicle, and the fornix link this anterior system and therefore constitute the conceptual and emotional scaffolding. The role of the hippocampus, located at the crossroad of these paths, would be to bind multimodal contents - including verbal cues (Barry \& Maguire, 2019; Moscovitch et al., 2016) - both during encoding and retrieval (de VanssayMaigne et al., 2011; Diana et al., 2007), allowing for unified memories. Although examined mainly in isolation, the substrates and processing pathways highlighted in contemporary language and declarative memory models suggest an inevitable intertwining (Figure 1) that we aim to describe and formalize in the following sections.

-Insert Figure 1 here

\subsection{Beyond the independence of language and memory}

\subsubsection{Overlap of fundamental dimensions}

Language and declarative memory overlap. They can be considered part of a larger and interactive system, but their interaction to support unified behavior remains undetermined. The "common ground" introduced by Clark and Marshall (Clark \& Marshall, 1981) as a basis of interpersonal interaction via language is a tangible cognitive example for explaining the unified view of language and declarative memory. Individuals communicate by relying on the shared set of beliefs, ideas, and knowledge while also making assumptions about the interlocutors' perspectives. These elements constitute the "common ground", a concept close to mentalization (Premack \& Woodruff, 1978). According to the resonance-based theory of common ground, Horton (Horton, 2007) proposed that working memory information, such as current discourse, resonates with long-term memory information (such as the partner's 
identity, traits and attributes, past events or similar discourse) in a fast and automatic fashion (Brown-Schmidt \& Duff, 2016), to ensure a relevant and contextually appropriate conversation.

What may be the neurocognitive foundation of common ground? First, the discrete cognitive constructs conventionally ascribed to language and declarative memory are interconnected and exhibit some entanglement (Box 1). Second, the functional brain representations associated with each of these constructs reveal a complex of three major fundamental components that may capture the language-memory continuum (Figure 2A). Finally, the arrangement of cognitive constructs within these latent canonical components further provides insight into the dimensions' composition, specificities, and characteristics (Figure 2B) and thus offers both a global and composite view (Figure $2 \mathrm{C}$ ) of the common ground.

From a neurocognitive point of view, the first latent dimension - which we propose to call "Embodiment" (EMB) - mainly encompasses aspects related to speech perception, phonology, articulation, and syntax. In addition, and even if to a lesser extent, working memory and comprehension saturate this dimension. This suggests that EMB comprises processes related to the externalization of verbalizable outputs, implying "spell-out" and sensory inputs influencing all cognitive processes and the outputs (cognitively embodied). EMB may involve perceptuo-motor information processing operations (Giraud \& Poeppel, 2012; Poeppel \& Assaneo, 2020, for an example of computational principles associated with speech perception) - selection/detection, segmentation, concatenation/linearization, conjunctive binding. To simplify and caricatured, EMB could proceed at the computational level as an active cognitive "Receiver-Transmitter".

The second dimension is represented primarily by verbal working memory and comprehension. This component is also more broadly related to articulation, phonology, syntax, associative memory, and lexical access/retrieval. Thus, it could refer to the controlled assembly of elementary operations allowing to transform a verbal input actively into an elaborated and appropriate verbal output (i.e., the accurate mapping between meaning and sounds or, conversely, between sounds and meaning; between word and signification or between sentences/discourse and meaning, depending on the level of processing). Concretely, incremental binding, monitoring, evaluation, or (error-)prediction operations can be engaged as active inference algorithms (i.e., predicting future states according to the trajectory defined by a given policy; Parr \& Friston, 2017). We propose to call this dimension "Formulation" (FORM) because "formulation" relates, in a broad sense, to the way elements or a mixture of elements are manipulated and assembled for a specific purpose. Thus, FORM can be roughly characterized as a "Controller-Manager". In agreement with Levelt, the formulation can be understood at the computational level as the action of turning the message into (linguistic) representation. However, if Levelt distinguishes the "formulator" (speech production component) from the "parser" (speech comprehension system; Levelt, 1989, 1993), FORM would here engage operations common to language production and comprehension (Figure 2B). This is consistent with neuroimaging studies demonstrating a neat overlap between natural language production and comprehension's functional maps (e.g., Silbert et al., 2014).

The third dimension covers neurocognitive aspects related to language comprehension, associative memory, lexical access/retrieval, verbal semantic, episodic, and working memories. We have named this dimension "Internalization" (INT) as it includes computations to build and maintain mental, conceptual, and multimodal representations. INT can therefore be described as a "Transformer-Associative" computational component (again in a simplistic way). The operations underlying INT include, for instance, abstraction/dimensionality 
reduction, multimodal/relational binding, pattern separation/completion, and replay (Cowell et al., 2019).

To summarize the findings and postulates, there is a substantial overlap between the languagememory cognitive constructs and their brain representations, revealing an underlying continuum of three main latent dimensions (Figure 2). The detected dimensions (EMB-FORMINT) may share some features with those of previously described models in the context of functional language modeling (Berwick et al., 2013 for the "basic design of language" model; or Hagoort, 2013, 2014, 2017 for the MUC model, in particular). However, the modeling we provide emphasizes no component that can be described by a (single) cognitive function or subfunction (e.g., memory, syntax, or executive functions). The nature of the canonical detected dimensions imposes instead a new ontology based on overlaps. Some constructs, such as the working memory outlined above, are positioned at the intersection of the tripartite EMB-FORM-INT complex, which could confirm their central place in the common ground and, by extension, in the language-memory unification process. Nevertheless, it is necessary to go beyond this cognitive framework to identify the specific and biologically plausible building blocks in concern. If so far, we have referred, in the Marr's spirit (Marr, 1982), the putative (1) computational goals and (2) algorithmic operations involved in such interactive components; the (3) neural implementation or neurobiological relevance of these dimensions as well as their interactions will be discussed in the following sections.

-Insert Figure 2 here-

\subsubsection{Shared substrate}

Verbal communication requires a multitude of intra-language aspects (e.g., knowledge about sounds, grammar, word meaning, and general world knowledge; Hogan et al., 2014) and extralanguage aspects (e.g., attention monitoring and making inferences in addition to long term memory retrieval; Kintsch \& Kintsch, 2005), allowing for the flexible organization of multimodal information into a single meaningful representation. This highly interactive cognitive structure requires an adequate and specialized anatomo-functional brain substrate.

Few studies have explored the common substrates of language and long-term declarative memory, especially episodic memory (Duff \& Brown-Schmidt, 2012). The examination of the meta-analytic functional maps for terms related to language and memory (provided by Neurosynth and based on hundreds of fMRI studies; Yarkoni et al., 2011) yields a first approximation of the relationships between these two functions and their associated constructs (Figure 3). In agreement with the prior literature, the functional meta-analysis emphasizes the concept of working memory at the intersection of language-memory functions (Figure 3B). Common brain regions and fascicles have been previously associated with executive processes (Acheson et al., 2010) and mostly correspond to the cerebral representation of working memory (Eriksson et al., 2015). However, neocortical temporal and hippocampal regions and ventral and mesial fascicles classically described for their involvement in episodic and semantic memory (Figure 1) are also considered "shared areas". This is in line with some recent proposals that include mesial regions at the interface between language and memory (e.g., Brown-Schmidt \& Duff, 2016; Covington \& Duff, 2016) and indicate that the interactions are complex and not limited to the verbal working memory component alone (Figure 3 ). 
These common territories express a certain degree of functional cooperation or joint neural integration. Nevertheless, despite its great interest, the conceptualization of interdependencies between different cognitive "domains" is not yet fully established in the current literature. Since connectivity analysis explicitly "bridges localizationist and distributed theories of brain function" (Medaglia \& Bassett, 2018), we formalize here their interplay through the lens of networks to propose new conceptions. This perspective expands Hagoort's proposal of "networks of interest" (NOIs; Hagoort, 2014) beyond language and the perisylvian network.

\subsubsection{Synergistic networks interactions}

Modern accounts of the functional connectome organization at various space-time scales have described hierarchical networks, such as the Default Mode Network (DMN; Mazoyer et al., 2001; Raichle et al., 2001), whose activity is strongly coupled to the resting state. These networks are composed of several key regions that confirm the "multifocal" conception of brain functioning proposed by Mesulam in the early 1990s and which "gives rise to brain-behavior relationships that are both localized and distributed" (Mesulam, 1990). The regions involved in these networks are essential for certain types of cognitive state or processing (e.g., in visual perception or executive control; Rosazza \& Minati, 2011), thus making it possible to attribute a "cognitive" dimension to functional networks.

Intrinsic functional networks follow an ordered sequence of attributes (i.e., properties such as the degree or the nature of functional integration of the representations). The cortical organization extends along a functional continuum that spans from unimodal sensorimotor areas to transmodal associative regions of the DMN, passing by attention and executive areas (Margulies et al., 2016; G1 in Figure 4B). Unimodal networks are more modular and segregated. More specifically, the organization of the connectivity of sensorimotor, visual, and auditory networks follows topographic representations across adjacent cortices, favoring hierarchical serial processing of information (in terms of complexity). Conversely, the connectivity profiles of transmodal executive-associative networks, such as the DMN, the fronto-parietal control network (FPN), or even the ventral salience and dorsal attentional networks (SAL and DAN, respectively; Yeo et al., 2011) consist in "long-range" networks linking spatially distant regions (and not close neighbors). Overall, modern conceptions described the general neurocognitive functioning as hybrid architecture. Such architecture will combine modularity and interconnectivity and balance between integration/segregation of networks, facilitating an efficient transfer of information (Achard \& Bullmore, 2007). The dynamical synchronization between networks (or multi-network state or meta-systems (Cocchi et al., 2013) supports a "hierarchical encapsulation" involving nested networks within networks (Hilgetag \& Goulas, 2020). These complex configurations of inter-networks coupling may be at the origin of interactions between cognitive functions (Herbet \& Duffau, 2020). Therefore, the re-evaluation of language and declarative memory under this prism brings new grounds for a less fragmented view of the functioning and addresses how the multiple facets are synergistically embedded in an adaptive, harmonious, and unified process. 


\section{Towards a unified view of language and memory}

\subsection{LUM formalism and main principles}

To account for the dynamic exchanges, we conceive language and memory on a continuum composed of the three fundamental dimensions (EMB-FORM-INT; Figure 2C) in interactions (Figure 4A). The proposed interactive neurocognitive new framework "LUM" (for Language/union/Memory) adopts a neuro-centric and connectivity-based vision to reconcile the language-memory relations' cognitive manifestations within a biologically plausible structure. We argue that language and memory relationships cannot be described by a unique stationary network but mainly through a network's interactions' triplet. Specifically, the SAL, FPN, and DMN networks appear to be primarily engaged in the EMB, FORM, and INT latent dimensions that we propose, respectively. This proposal relies on direct evidence from the observation of the functional connectome (Figure 4B) and the literature's experimental findings (e.g., Braga et al., 2020; Wang et al., 2019 for recent research). Box 2 summarizes the principles that govern the LUM framework.

LUM engages large dynamic configurations and reconfigurations of online/offline transient states. This framework argues that these networks' global and regional functional dynamics are crucial to describe language-memory behaviors. At the global level, the dialogues between the networks form a "ballet of networks", underpinning the inter-function relationships (Hagoort, 2014). Key regions (hubs) may have a functional architecture that actively sustains and coordinates networks at a local level. The synergy of these global and local dynamics orchestrates and supports language-memory operations.

-Insert Figure 4 here-

\subsection{LUM dimensions and functional modules}

While there is a continuum between these SAL-FPN-DMN associative networks, they all carry their functional characteristics. Their anatomical topology is independent, with abrupt spatial transitions between the connectivity patterns (Yeo et al., 2011), aligning with the LUM latent dimensions formalized (at least partially) distinct modules.

\subsubsection{SAL, the cognitive "Receiver-Transmitter" (Embodiment)}

Accompanied by information from the sensory-motor network (SMN, including mainly motor and auditory-perceptive networks), the ventral attentional network [also called salience network (SAL)] presents the functional properties to support the Embodiment dimension (EMB). SAL is triggered by the salience of external stimuli and the detection of relevant external targets, thus managing the attention's top-down and "exogenous" reorientation (Matthen, 2005). Linked to sensory awareness, SAL filters the environment, allowing the rapid and active-controlled integration of exteroceptive information, and providing relevant information in working memory (Parr \& Friston, 2017). Therefore, the SAL network has optimal intrinsic properties for supporting the cognitive reception-transmission dimension of auditory and verbal information (e.g., for active listening or controlled externalization).

\subsubsection{FPN, the "Controller-Manager" (Formulation)}

The Formulation dimension (FORM)'s primary function is the organization, development, and maintenance of verbal representations. It would be underpinned by a top-down controlled 
network (sometimes called Multiple Demand Network: MDN or Central Executive Network: CEN; Doucet et al., 2019). More precisely, the fronto-parietal control network (FPN), especially lateralized in the left hemisphere, could be involved in a privileged way. FPN is observed in verbal and autobiographical planning (Benedek et al., 2016) or semantic control (Xu et al., 2017). In general, this network supports cognition and goal-oriented behaviors and is linked to verbal working memory and fluid intelligence (Assem et al., 2020). Thus, the fronto-parietal network properties could facilitate managing the various executive operations required for verbalization and declarative memory.

\subsubsection{DMN, the "Transformer-Associative" (Internalization)}

Processes related to the Internalization dimension (INT) mainly involve the highly integrative default network (DMN). Indeed, the DMN has previously been associated with internal and offline attention (Buckner et al., 2008), allowing the elaboration of self-generated, introspective thinking built on memory content (Andrews-Hanna et al., 2014; Konishi et al., 2015). The DMN would also be engaged in mental imagery and, therefore, episodic memories. It would allow temporal, spatial, and content distortion, offering the possibility to imagine neverexperienced situations and produce complex thoughts (Binder \& Desai, 2011; Boyer, 2008). It could thus underlie the "mental time travel" involved in episodic memory (Humphreys \& Lambon Ralph, 2015; Lau et al., 2013; Raichle, 2015; Wang et al., 2020), especially when coupled to the limbic system (Jeong et al., 2015; McCormick et al., 2014). Several studies have shown its involvement in semantic declarative memory's various cognitive tasks, facilitating the resonance between environmental characteristics and those derived from similar prior knowledge and states (Binder \& Desai, 2011; Constantinescu et al., 2016). As a multimodal experiential system (Xu et al., 2017), the highly integrative characteristics of DMN appear indispensable to support the internalization dimension of elaborated representations (INT). The DMN activity would be "prosocial" and has probably adapted to the evolution of language. In humans, the DMN collects and facilitates auditory information integrations (Simony et al., 2016) and promotes social content (Dohmatob et al., 2020; Krienen et al., 2010).

\subsection{LUM interactions: global and local dynamics}

\subsubsection{Non-stationary global dynamics of networks}

We can assume that a "networks ballet", happening through SAL-FPN-DMN transitions and dynamical synchronizations (Figure 4), can actively and synergistically support the LUM cognitive states and the "common ground" mentioned in section 2.3.1. Some research on language or semantic memory highlights a synergy of different network components (multinetwork states; Braga et al., 2020; Gordon et al., 2020; Hagoort, 2019; Ji et al., 2019; X. Wang et al., 2020). Networks maintain complex mutual relationships with each other, controlling other networks' functions, others acting in interaction (Buckner et al., 2013). Recently, Cocuzza et al. (Cocuzza et al., 2020) have proposed that cognitive task control can be performed dynamically by high-level controlled networks but differently. SAL would play the role of "flexible switcher" and the FPN would be a "flexible coordinator". More precisely, FPN actively coordinates other regions' activities to obtain information relevant to the goal to be achieved. It thus allows cooperation between networks initially considered as antagonistic (e.g., DMN-SAL or DMN-DAN synchronization; Spreng et al., 2013; Wang et al., 2020). Unlike FPN, SAL dissolves transiently (intra-network decoupling) to lend processing resources to other networks pertinent to the objectives. This transition is described as a dynamic "switch", allowing to initiate the transition from an interoceptive state (DMN) to a goal-oriented state 
(FPN and FPN-DMN coupling, involved in phasic vigilance; Cocchi et al., 2013). Within the LUM framework, FPN and SAL's interaction can control processing towards the most critical external cues in a conversation. In interaction with the DMN, FPN can actively extract relevant internal information from previous experience and knowledge.

\subsubsection{Local dynamics of integrative hubs}

By attempting to locate the language within the organization of intrinsic networks, DiNicola et al. (2020) showed that language-associated regions exhibit a similar pattern of juxtapositions of several networks. These cortical sites, located at the crossroads of the leading networks, thus present essential properties to act as "connector hubs" that are core regions able to integrate information from the different networks locally (see also Braga et al., 2013 for a description of the local multi-networks echo phenomenon). Among these convergence areas, the inferior frontal gyrus (IFG complex) follows a SAL-FPN-DMN gradient during the transition from pars opercularis to pars orbitalis (Figure $4 \mathrm{C}$ ). The IFG complex could functionally and gradually integrate phonological, syntactic, and semantic representations.

The associative role of the IFG is concordant with its previously reported role in the construction of conceptual and syntactical hierarchies (Goucha et al., 2017). More than the region per se, the connections to and from the IFG confer this role of assembling linguistic representations. These observations fit with the MUC model proposed by Hagoort (Hagoort, 2016), describing a tripartite "Memory-Unification-Control" hierarchy within the IFG, maintained by structural connections with other areas of functional convergence such as the temporoparietal junction (TPJ) or the posterior part of the mean temporal gyrus (pMTG). Indeed, anatomically, these connector hubs receive the projection of crucial structural pathways (e.g., the arcuate and branches II-III of the superior longitudinal fascicle; Figure 4C) and thus constitute essential convergence zones for inter-network integration. Similar local gradients exist in the insula, the supramarginal and angular gyrus, the posterior upper/mid temporal gyrus, the supplementary motor area, the dorsolateral prefrontal cortex, the cerebellum, and the basal ganglia. These local integrators could serve as interfaces to interconnect the different LUM dimensions by manipulating external information and internal mental representations. Their role could be particularly crucial when the demand for internetwork connection is reinforced, e.g., during online activity.

Finally, the role of "peripheral hubs" that strengthen intra-network connections is also central (for instance, Dohmatob et al., 2020) to clarify the offline functional specialization of the main hubs belonging to the $\mathrm{DMN}$ ). One of these peripheral hubs is the hippocampus linking information from the anterior-posterior DMN regions at rest (Barnett et al., 2020). Due to its intrinsic properties and connectivity (see the next section for more details), the hippocampus would have an active role in relational binding (i.e., binding multimodal information; Jonin et al., 2019) using high-fidelity information coming from the other DMN regions.

\section{Relevance and future directions}

\subsection{Functional specialization}

The LUM interactive model provides benchmarks to apprehend interactions between functions, specifically language and declarative memory. The formalization of these broader (and interactive) configurations shows that the cognitive-centric nomenclature classically used to 
account for these behaviors (Box 1) is not adequate. Cognitive processes and psychological constructs are traditionally identified from observable and salient behaviors and are hybrid concepts with blurred definable (Cowell et al., 2019; Figure 2A), which limit our understanding of brain-behavior relationships (Anderson, 2011). It would now be advisable to provide a new vocabulary to circumscribed cerebral mechanisms rather than looking for cerebral mechanisms behind psychological constructs (see Buzsáki, 2020, for an opinion review on the topic).

Neurocognitive operations or neuroperations describing latent neuro-mechanisms (as opposed to constructs resulting from observable processes) may be good candidates. Operations indeed reflect the brain's modus operandi or how the brain manipulates information to generate mental representations and behaviors (i.e., neuro-computational manipulations). They thus enable to approach brain-behavior relationships with a neuro-centric and a connectivity-based vision, which is necessary for the study of interactions not directly observable by essence. It would explain functional specialization from a different perspective than the one used so far. Given that a specific brain region is often involved in a large variety of behaviors (functional polyhedron; Genon et al., 2018), it becomes difficult to infer the functional specialization without an understanding of the core neuro-computational function(s).

In linguistics and for example, the latent operation "Merge" has been proposed to account for the combinatorial mechanism that brings elements together to form an unordered set of higherorder items and would thus be the crucial operation behind what we called syntax (Chomsky, 1995; Goucha et al., 2017, for a neurocognitive description). "Labeling," by operating classification of verbal information into an ordered set of elements here enables the creation of categories considered as a critical characteristic of human communication (Boeckx \& BenítezBurraco, 2014) and is proposed as a neuro-relevant operation (Benítez-Burraco \& Murphy, 2019; Murphy, 2015a, 2015b, for a model of brain dynamics as well as an algorithmic description). In the memory field, neurocognitive operations such as pattern separation, pattern completion, and replay are key neuro-mechanisms of encoding, retrieval, and active maintenance, respectively (Cowell et al., 2019; Pidgeon \& Morcom, 2016; Yassa \& Stark, 2011). Memory is an integral part of each cortical zone's functioning (see Hasson et al., 2015 and their hierarchical conceptualization of memory). For example, a conjunctive binding, by the cooccurrence of perceptive attributes (Jonin et al., 2019), involved in visual recognition also requires pattern completion operations. The property of relational binding, leading to multimodal and unified representation, emerges only collectively because of interactions between different brain circuits.

This "networked" conceptualization of functional specialization as proposed in the LUM model does not mean that all regions have a similar neuro-functional architecture or equal contribution. For example, the prefrontal cortex involved in FPN may be slightly biased in favor of control and monitoring operations. When actively engaged in verbal information processing, the IFG (pars opercularis in particular) could be an essential contributor to assembly operations (Merge; Zaccarella \& Friederici, 2015). The hippocampus architecture is conducive to active links between multimodal information (Ekstrom \& Yonelinas, 2020; Figure 5 for details). Together and integrated into a vast network, these regions actively link different elements, leading to an increasingly rich representational content. The global and local dynamics features put forward in the LUM framework can explain how such neurocognitive operations dynamically operate to unify language and memory contents into an internal multimodal representation (Figure 5 for a demonstration). However, a more systematic and precise inventory of neurocognitive operations that may represent the cornerstone of 
understanding the interdependencies between different cognitive "domains" remains necessary. It thus constitutes one of the most important future challenges of cognitive neuroscience.

-Insert Figure 5 here

\subsection{LUM in clinical neuroscience}

The LUM model's fundamental specificity goes beyond the modular cognition vision by focusing on network dynamics and interactions. Doing so can provide an interesting angle of view on language and memory processes in healthy brains (e.g., development, plasticity due to learning, functional specialization or aging). It can also account for these functions' disturbances after brain injury (e.g., stroke, primary progressive aphasia, mild cognitive impairment, hippocampal hypoxia, epilepsy, or certain psychogenic disorders). Neuroplasticity (adaptive or maladaptive) does not follow random laws. For instance, unmasking a set of latent sub-circuits can take over operations sufficiently close to those normally required (Collignon et al., 2011). In this context, the simplified SAL-FPN-DMN network space of the LUM framework provides a taxonomy and guidance of where and how language and memory malfunctioning occur (see Figure 6 for an example of application in focal epilepsy). Rather than listing observable symptoms, the LUM connectivity-based and interactive model provides guidelines for evaluating and interpreting patients' dysfunctions. The framework allows reconsidering the pathology as affecting mechanisms that transcend the cognitive functions traditionally described.

LUM dysfunctions may mainly result from the impairment of a particular network. For example, post-stroke cognitive dysfunctions tend to occur in the context of disruption of a specialized network, affecting preferentially areas sharing similar connectivity patterns of those of the lesion zone (intra-network dysfunction; e.g., Bayrak et al., 2019). This is in line with previous studies showing that the diaschisis preferentially affects functional connectivity between related areas within a given network (Baldassarre et al., 2016; Ovadia-Caro et al., 2013; Siegel et al., 2016). Other pathologies, such as mild cognitive impairment (MCI), could also primarily affect intra-network connectivity. The most frequently reported finding is decreased connectivity within the DMN (Krajcovicova et al., 2014). This hypothesis of DMN modifications is consistent with findings that patients with MCI often present amnestic MCI and show poor performances for naming, fluency, word definition, spontaneous descriptions (Ahmed et al., 2008; Oulhaj et al., 2009), all involving representations embedded in the INT dimension of the LUM model. Depending on the progression of the pathology (e.g., conversion from MCI to Alzheimer's disease), disruption may become more significant. They can impact other networks and inter-network integration (Badhwar et al., 2017; Chand et al., 2017, for a systematic review and a meta-analysis), altering cognition more widely. LUM perturbations could also be due to abnormal modulation of inter-networks dialogues. Even though this hypothesis has not been directly tested, possible abnormal FPN-DMN (or FORM-INT) synchrony, in particular, could also be the cause of disorders such as psychogenic amnesia, for instance. The mechanism of involuntarily repressed memories in patients suffering from 
dissociative amnesia is reflected by the over-activation of prefrontal cortices leading to an under-activation of the hippocampal complex (Kikuchi et al., 2010).

Concerning local dynamics, several lines of evidence have shown that central hubs are highly prone to damage (Fornito et al., 2015). When neuropathology affects language-memory areas of convergence (damage to cortical connectors), as in certain forms of stroke or primary progressive aphasia (PPA), it results in a profound perturbation of inter-networks (LUM) integration and global modularity (Gratton et al., 2012; Power et al., 2013). Lesions or malfunctions of these areas lead to more severe deficits (e.g., Wilson et al., 2012), inducing symptoms of so-called Broca's or Wernicke's aphasia that could be translated, in computational terms, by interference in critical operations such as "Merge" or "Label".

In addition to acute injury, developmental neuropathology is also a privileged setting for identifying the resilience of the LUM system to cope with the constraints imposed by an atypical brain configuration. For example, dissociations between language and memory have been reported in amnesic patients with neonatal hypoxia and bilateral hippocampi damage (Vargha-Khadem et al., 1997; Vargha-Khadem \& Cacucci, 2021, for a recent commentary). Despite apparent "autonetic" amnesia, these children who grew up without functional hippocampi show vast preservation of the verbal "semantic" learning and productioncomprehension language abilities (Gadian et al., 2000). However, similar damage occurring in adulthood can lead to quantifiable disorders of naming or the social use of language (Duff et al., 2009; Hilverman \& Duff, 2021). The idiosyncratic interdependencies and equilibria specific to an early atypical brain configuration have yet to be specified. In the LUM framework, this effort will help to understand how language-memory harmony can remain (relatively) preserved despite the disruption of essential operations as here, multimodal binding.

The timing, the type, and the extent of damage condition the degree of functional specialization and cognitive efficiency. The compensatory potential of the (developing) brain, however, can be highly impressive. Kliemann and colleagues found that childhood hemispherectomy patients may present remarkable preservation of their cognitive abilities, including language and memory (Kliemann et al., 2019). Those patients, despite the absence of one hemisphere and thus of homotopic organization and interconnection, have nonetheless developed a close to typical pattern of functional connectivity in the remaining hemisphere, maintaining the segregation and specificity of specialized and intrinsic networks (see also Tyszka et al., 2011 for similar observations in congenital agenesis of corpus callosum). The preservation of the global and local stereotypic LUM connectivity gradient thus appears to be an essential predicate for harmonious language and memory development. Other factors or neuroplasticity mechanisms such as degeneracy or pluripotentiality (Friston \& Price, 2003; Noppeney et al., 2004) make the structure-function relationships complex need to be explored in the study of LUM disorders.

Overall, the application potential of the model is multiple. A focus on how LUM networks/brain regions are functionally modulated can bring new working hypotheses on the origin of (mal)adaptive plasticity, help diagnose clinical targets, and support the refinement of existing nosology. Conversely, testing the LUM model in various conditions (developmental/longitudinal, before/after neurosurgery, before/after cognitive rehabilitation) and pathologies represent an eminent source for enriching the model by helping, for example, to inventory LUM neurocognitive operations and associated critical regions/connections/dialogues. 


\subsection{Cerebello-subcortico-cortical synchrony}

Although very comprehensive and detailed, the current language models (Duffau et al., 2014; Hickok \& Poeppel, 2007; Indefrey, 2011; Price, 2012) do not incorporate mesial temporal structures that contribute to language processing (Bonhage et al., 2015; Hamamé et al., 2014; Piai et al., 2016). Although still under debate, the hippocampus's role in processing semantic memory and, more broadly, of language could be more important than previously considered (Covington \& Duff, 2016; Duff et al., 2020; Spiers, 2020) and should be studied further LUM context.

Moreover, the central place given to subcortical and cerebellar structures in higher-order cognitive functions, such as language and memory, remains relatively recent, probably owing to the implicit legacy left by the theory that the subcortical regions underlie primitive functions. Basal ganglia and striatum would be involved in sequencing (Leventhal et al., 2012) necessary for the working memory system (Parr \& Friston, 2017) and in respect to language processing in the formulation of "syntactic complexity" (Santi et al., 2015). The parallel loops between the striatal complex and cortical region also engage the thalamus, a highly integrative hub of multimodal information, crucial for the language and long-term memory (Wolff \& Vann, 2019; Figure 5B). Its anterior part, strongly linked to the hippocampus, would be notably engaged for in-memory recollection processes, constituting a "gateway to mental representations" (Aggleton et al., 2010; Johnson \& Knight, 2015 for evidence coming from intracranial EEG). Finally, the cerebellum (or "little brain") is a complex structure whose involvement goes beyond language production's motor aspects. More precisely, Guell and Schmahmann (2020) have recently dissected its functional anatomy and have shown a specific functional ordering involving gradually motor, attentional/executive (mainly represented by working memory), default-mode (verbal task as story listening) aspects of cognition from the anterior to the posterior part of the cerebellum. The change from static to a dynamic view of cognition sheds light on the importance of these structures' roles in higher cognitive functions (Benítez-Burraco \& Murphy, 2019). These regions' systematic functional connectivity investigation will ensure a more precise delineation of their involvement and specialization, which will enrich the current model.

\subsection{Leveraging advances in functional connectomics}

Advances and methodological transfers in network sciences make it possible to propose new modeling to complex layouts (Bassett et al., 2018) and, in particular, to inter-function configurations (here LUM for language-memory relations). The multitude of new parameters offered for the study of networks (Lynn \& Bassett, 2019) makes it possible to define, ever more precisely, the neuro-functional properties of certain cerebral regions or networks (e.g., reflecting integration/segregation, flexibility/allegiance, hierarchies/heterarchies relationships). However, despite rapid progress, there is still no consensus on the number of network components that optimally describe brain function. The main networks we described in the LUM model could be effectively decomposed into several sub-networks or partitions (Akiki \& Abdallah, 2019; Gordon et al., 2020; Yeo et al., 2011), and their implementation, therefore, represents one of the prospects for refining the model. 
On the other hand, if functional connectivity has so far given a central place to nodes/brain regions, a new methodological approach based on the study of the properties of the connections themselves (an edge-centric approach; Faskowitz et al., 2020; Uddin, 2020) is an exciting perspective of added value for interactive models and framework. In addition, functional connectivity is now directly approaching dynamically (time-varying network representations; Medaglia \& Bassett, 2018). Dynamical functional connectivity allows a better approach to the notion of "cognitive state" and thus provides crucial insights for investigating transient configurations and neurocognitive operations. In the near future, interactive models, such as the LUM model, will significantly benefit from developing and applying dynamical systems theory methods (see, in this perspective, Forseth et al., 2021). Moreover, the improvement of dynamical connectivity will also benefit the study of effective connectivity, causal relations between temporal signals, which should also greatly help clarify the "how" of language memory operations are performed by the brain and their failure. Finally, computational methods and artificial neural networks are also promising tools that, coupled with functional brain markers, can allow a better understanding of the computations and algorithms involved in natural language processing as well as their neural implementation (e.g., Goldstein et al., 2021; Jain \& Huth, 2018, for two examples on word prediction in natural context; Martin, 2020, for a proposed architecture of the hierarchical and compositional structure of language, based on neurobiological and neurocomputational modeling evidence).

\section{Conclusions}

Recent language or memory models emerged in recent years to improve our knowledge of neurocognitive processes and substrates underpinning these functions. The current paradigm tends to enrich these models and contribute parsimoniously to unify our vision of cognitive functioning, which, at this moment, remains fragmented. There is still a limited number of theories depicting relationships and dynamics between cognitive functions. We proposed the LUM model to address this issue and integrate the notable research findings with the more recent ones by considering the network theory perspective to provide useful benchmarks. Due to its interactive framework, LUM proposes a new taxonomy (EMB-FORM-INT neurobiologically supported by SAL-FPN-DMN) that can help understand normal development and aging and cognitive language and memory dysfunctions in patients with neuropsychiatric disorders. The model proposed goes beyond the traditional cognitive-centric approach. It is scalable and can thus be adapted to other cognitive domains. We present only a first theoretical step toward models that could design and help better understand the interactive dynamics of cognitive functions and meta-functions. 


\section{References}

Aboitiz, F. (2012). Gestures, Vocalizations, and Memory in Language Origins. Frontiers in Evolutionary Neuroscience, 4. https://doi.org/10.3389/fnevo.2012.00002

Aboitiz, F., Aboitiz, S., \& García, R. R. (2010). The Phonological Loop: A Key Innovation in Human Evolution. Current Anthropology, 51(S1), S55-S65. https://doi.org/10.1086/650525

Achard, S., \& Bullmore, E. (2007). Efficiency and Cost of Economical Brain Functional Networks. PLOS Computational Biology, 3(2), e17. https://doi.org/10.1371/journal.pcbi.0030017

Acheson, D. J., Hamidi, M., Binder, J. R., \& Postle, B. R. (2010). A Common Neural Substrate for Language Production and Verbal Working Memory. Journal of Cognitive Neuroscience, 23(6), 1358-1367. https://doi.org/10.1162/jocn.2010.21519

Aggleton, J. P., O'Mara, S. M., Vann, S. D., Wright, N. F., Tsanov, M., \& Erichsen, J. T. (2010). Hippocampal-anterior thalamic pathways for memory: Uncovering a network of direct and indirect actions: Hippocampal-thalamic pathways for memory. European Journal of Neuroscience, 31(12), 2292-2307. https://doi.org/10.1111/j.1460-9568.2010.07251.x

Ahmed, S., Arnold, R., Thompson, S. A., Graham, K. S., \& Hodges, J. R. (2008). Naming of objects, faces and buildings in mild cognitive impairment. Cortex, 44(6), 746-752. https://doi.org/10.1016/j.cortex.2007.02.002

Akiki, T. J., \& Abdallah, C. G. (2019). Determining the Hierarchical Architecture of the Human Brain Using Subject-Level Clustering of Functional Networks. Scientific Reports, 9(1), 19290. https://doi.org/10.1038/s41598-019-55738-y

Allone, C., Lo Buono, V., Corallo, F., Pisani, L. R., Pollicino, P., Bramanti, P., \& Marino, S. (2017). Neuroimaging and cognitive functions in temporal lobe epilepsy: A review of the literature. Journal of the Neurological Sciences, 381, 7-15. https://doi.org/10.1016/j.jns.2017.08.007

Anderson, B. (2011). There is no Such Thing as Attention. Frontiers in Psychology, 2. https://doi.org/10.3389/fpsyg.2011.00246

Andrews-Hanna, J. R., Smallwood, J., \& Spreng, R. N. (2014). The default network and self-generated thought: Component processes, dynamic control, and clinical relevance. Annals of the New York Academy of Sciences, 1316(1), 29-52. https://doi.org/10.1111/nyas.12360

Assem, M., Blank, I. A., Mineroff, Z., Ademoğlu, A., \& Fedorenko, E. (2020). Activity in the frontoparietal multiple-demand network is robustly associated with individual differences in working memory and fluid intelligence. Cortex, 131, 1-16. https://doi.org/10.1016/j.cortex.2020.06.013

Badhwar, A., Tam, A., Dansereau, C., Orban, P., Hoffstaedter, F., \& Bellec, P. (2017). Resting-state network dysfunction in Alzheimer's disease: A systematic review and meta-analysis. Alzheimer's \& Dementia: Diagnosis, Assessment \& Disease Monitoring, 8, 73-85. https://doi.org/10.1016/j.dadm.2017.03.007

Baldassano, C., Chen, J., Zadbood, A., Pillow, J. W., Hasson, U., \& Norman, K. A. (2017). Discovering Event Structure in Continuous Narrative Perception and Memory. Neuron, 95(3), 709-721.e5. https://doi.org/10.1016/j.neuron.2017.06.041

Baldassarre, A., Ramsey, L. E., Siegel, J. S., Shulman, G. L., \& Corbetta, M. (2016). Brain connectivity and neurological disorders after stroke: Current Opinion in Neurology, 29(6), 706-713. https://doi.org/10.1097/WCO.0000000000000396 
Banjac, S., Roger, E., Pichat, C., Cousin, E., Mosca, C., Lamalle, L., Krainik, A., Kahane, P., \& Baciu, M. (2021). Reconfiguration dynamics of a language-and-memory network in healthy participants and patients with temporal lobe epilepsy. NeuroImage. Clinical, 31, 102702. https://doi.org/10.1016/j.nicl.2021.102702

Barnett, A. J., Reilly, W., Dimsdale-Zucker, H., Mizrak, E., Reagh, Z., \& Ranganath, C. (2020, juin 10). Organization of cortico-hippocampal networks in the human brain. BioRxiv, 2020.06.09.142166.

Barry, D. N., \& Maguire, E. A. (2019). Remote Memory and the Hippocampus : A Constructive Critique. Trends in Cognitive Sciences, 23(2), 128-142. https://doi.org/10.1016/j.tics.2018.11.005

Bartha-Doering, L., \& Trinka, E. (2014). The interictal language profile in adult epilepsy. Epilepsia, 55(10), 1512-1525. https://doi.org/10.1111/epi.12743

Bassett, D. S., Zurn, P., \& Gold, J. I. (2018). On the nature and use of models in network neuroscience. Nature Reviews Neuroscience, 19(9), 566-578. https://doi.org/10.1038/s41583-018-0038-8

Bayrak, Ş., Khalil, A. A., Villringer, K., Fiebach, J. B., Villringer, A., Margulies, D. S., \& Ovadia-Caro, S. (2019). The impact of ischemic stroke on connectivity gradients. Neurolmage: Clinical, 24, 101947. https://doi.org/10.1016/j.nicl.2019.101947

Benedek, M., Jauk, E., Beaty, R. E., Fink, A., Koschutnig, K., \& Neubauer, A. C. (2016). Brain mechanisms associated with internally directed attention and self-generated thought. Scientific Reports, 6(1), 22959. https://doi.org/10.1038/srep22959

Benítez-Burraco, A., \& Murphy, E. (2016). The Oscillopathic Nature of Language Deficits in Autism: From Genes to Language Evolution. Frontiers in Human Neuroscience, 10, 120. https://doi.org/10.3389/fnhum.2016.00120

Benítez-Burraco, A., \& Murphy, E. (2019). Why Brain Oscillations Are Improving Our Understanding of Language. Frontiers in Behavioral Neuroscience, 13. https://doi.org/10.3389/fnbeh.2019.00190

Benke, T., Kuen, E., Schwarz, M., \& Walser, G. (2013). Proper name retrieval in temporal lobe epilepsy: Naming of famous faces and landmarks. Epilepsy \& Behavior, 27(2), 371-377. https://doi.org/10.1016/j.yebeh.2013.02.013

Berwick, R. C., Friederici, A. D., Chomsky, N., \& Bolhuis, J. J. (2013). Evolution, brain, and the nature of language. Trends in Cognitive Sciences, 17(2), 89-98. https://doi.org/10.1016/j.tics.2012.12.002

Binder, J. R., \& Desai, R. H. (2011). The neurobiology of semantic memory. Trends in Cognitive Sciences, 15(11), 527-536. https://doi.org/10.1016/j.tics.2011.10.001

Bloom, P. (2000). How Children Learn the Meanings of Words. MIT Press.

Boeckx, C. A., \& Benítez-Burraco, A. (2014). The shape of the human language-ready brain. Frontiers in Psychology, 5, 282. https://doi.org/10.3389/fpsyg.2014.00282

Bolhuis, J. J., Tattersall, I., Chomsky, N., \& Berwick, R. C. (2014). How Could Language Have Evolved? PLoS Biol, 12(8), e1001934. https://doi.org/10.1371/journal.pbio.1001934

Bonhage, C. E., Mueller, J. L., Friederici, A. D., \& Fiebach, C. J. (2015). Combined eye tracking and fMRI reveals neural basis of linguistic predictions during sentence comprehension. Cortex, 68, 33-47. https://doi.org/10.1016/j.cortex.2015.04.011

Boyer, P. (2008). Evolutionary economics of mental time travel? Trends in Cognitive Sciences, 12(6), 219-224. https://doi.org/10.1016/j.tics.2008.03.003 
Braga, R. M., DiNicola, L. M., Becker, H. C., \& Buckner, R. L. (2020). Situating the left-lateralized language network in the broader organization of multiple specialized large-scale distributed networks. Journal of Neurophysiology, 124(5), 1415-1448. https://doi.org/10.1152/jn.00753.2019

Braga, R. M., Sharp, D. J., Leeson, C., Wise, R. J. S., \& Leech, R. (2013). Echoes of the Brain within Default Mode, Association, and Heteromodal Cortices. Journal of Neuroscience, 33(35), 14031-14039. https://doi.org/10.1523/JNEUROSCI.0570-13.2013

Broca, P. (1865). Sur le siège de la faculté du langage articulé. Bulletins de la Société d'anthropologie de Paris, 6(1), 377-393. https://doi.org/10.3406/bmsap.1865.9495

Brown-Schmidt, S., \& Duff, M. C. (2016). Memory and Common Ground Processes in Language Use. Top Cogn Sci, 8(4), 722-736. https://doi.org/10.1111/tops.12224

Buckner, R. L., Andrews-Hanna, J. R., \& Schacter, D. L. (2008). The brain's default network: Anatomy, function, and relevance to disease. In A. Kingstone \& M. B. Miller (Éds.), Annals of the New York Academy of Sciences: Vol. 1124. The year in cognitive neuroscience 2008 (p. 1-38). Blackwell Publishing. http://doi.wiley.com/10.1196/annals.1440.011

Buckner, R. L., Krienen, F. M., \& Yeo, B. T. T. (2013). Opportunities and limitations of intrinsic $\begin{array}{llll}\text { functional connectivity MRI. Nature Neuroscience, } & 16(7), \quad 832-837 .\end{array}$ https://doi.org/10.1038/nn.3423

Bullock, D., Takemura, H., Caiafa, C. F., Kitchell, L., McPherson, B., Caron, B., \& Pestilli, F. (2019). Associative white matter connecting the dorsal and ventral posterior human cortex. Brain Structure and Function, 224(8), 2631-2660. https://doi.org/10.1007/s00429-019-01907-8

Buzsáki, G. (2020). The Brain-Cognitive Behavior Problem: A Retrospective. eNeuro, 7(4). https://doi.org/10.1523/ENEURO.0069-20.2020

Campbell, K. L., \& Tyler, L. K. (2018). Language-related domain-specific and domain-general systems in the human brain. Current Opinion in Behavioral Sciences, 21, 132-137. https://doi.org/10.1016/j.cobeha.2018.04.008

Carruthers, P. (2013). Mindreading in Infancy. Mind \& Language, 141-172.

Carstairs-McCarthy, A. (1999). The origins of complex language: An inquiry into the evolutionary beginnings of sentences, syllables, and truth. Oxford University Press on Demand.

Castro, L. H., Silva, L. C. A. M., Adda, C. C., Banaskiwitz, N. H. C., Xavier, A. B., Jorge, C. L., Valerio, R. M., \& Nitrini, R. (2013). Low prevalence but high specificity of material-specific memory impairment in epilepsy associated with hippocampal sclerosis. Epilepsia, 54(10), 1735-1742. https://doi.org/10.1111/epi.12343

Catani, M., \& Dawson, M. S. (2017). Chapter 31-Language Processing, Development and Evolution. In P. M. Conn (Éd.), Conn's Translational Neuroscience (p. 679-692). Academic Press. https://doi.org/10.1016/B978-0-12-802381-5.00049-X

Chand, G. B., Wu, J., Hajjar, I., \& Qiu, D. (2017). Interactions of the Salience Network and Its Subsystems with the Default-Mode and the Central-Executive Networks in Normal Aging and Mild Cognitive Impairment. Brain Connectivity, 77), 401-412. https://doi.org/10.1089/brain.2017.0509

Chomsky, N. (1995). The Minimalist Program. MIT Press. 
Clark, H. H., \& Marshall, C. R. (1981). Definite Reference and Mutual Knowledge. In A. K. Joshi, B. L. Webber, \& I. A. Sag (Éds.), Elements of discourse understanding (p. 10-63). Cambridge University Press.

Cocchi, L., Zalesky, A., Fornito, A., \& Mattingley, J. B. (2013). Dynamic cooperation and competition between brain systems during cognitive control. Trends in Cognitive Sciences, 17(10), 493-501. https://doi.org/10.1016/j.tics.2013.08.006

Cocuzza, C. V., Ito, T., Schultz, D., Bassett, D. S., \& Cole, M. W. (2020). Flexible Coordinator and Switcher Hubs for Adaptive Task Control. Journal of Neuroscience, 40(36), 6949-6968. https://doi.org/10.1523/JNEUROSCI.2559-19.2020

Collignon, O., Champoux, F., Voss, P., \& Lepore, F. (2011). Sensory rehabilitation in the plastic brain. In Progress in Brain Research (Vol. 191, p. 211-231). Elsevier. https://doi.org/10.1016/B978-0444-53752-2.00003-5

Condret-Santi, V., Barragan-Jason, G., Valton, L., Denuelle, M., Curot, J., Nespoulous, J.-L., \& Barbeau, E. J. (2014). Object and proper name retrieval in temporal lobe epilepsy: A study of difficulties and latencies. Epilepsy Research, 108(10), 1825-1838. https://doi.org/10.1016/j.eplepsyres.2014.09.001

Constantinescu, A. O., O'Reilly, J. X., \& Behrens, T. E. J. (2016). Organizing conceptual knowledge in humans with a gridlike code. Science, 352(6292), 1464-1468. https://doi.org/10.1126/science.aaf0941

Conway, M. A., \& Loveday, C. (2015). Remembering, imagining, false memories \& personal meanings. Consciousness and Cognition, 33, 574-581. https://doi.org/10.1016/j.concog.2014.12.002

Cooper, R. A., \& Ritchey, M. (2020). Progression from Feature-Specific Brain Activity to Hippocampal Binding during Episodic Encoding. J. Neurosci., 40(8), 1701-1709. https://doi.org/10.1523/JNEUROSCI.1971-19.2019

Corballis, M. C. (2019). Minimalism and Evolution. Front. Commun., 4, 46. https://doi.org/10.3389/fcomm.2019.00046

Corkin, S. (2013). Permanent present tense: The unforgettable life of the amnesic patient, H. M. (p. xix, 364). Basic Books.

Covington, N. V., \& Duff, M. C. (2016). Expanding the Language Network: Direct Contributions from the Hippocampus. Trends in Cognitive Sciences, 20(12), 869-870. https://doi.org/10.1016/j.tics.2016.10.006

Cowell, R. A., Barense, M. D., \& Sadil, P. S. (2019). A Roadmap for Understanding Memory: Decomposing Cognitive Processes into Operations and Representations. ENeuro, 6(4), ENEURO.0122-19.2019. https://doi.org/10.1523/ENEURO.0122-19.2019

Danker, J. F., \& Anderson, J. R. (2010). The ghosts of brain states past: Remembering reactivates the brain regions engaged during encoding. Psychological Bulletin, 136(1), 87-102. https://doi.org/10.1037/a0017937

de Boer, B. (2011). Self-organization and language evolution. In K. R. Gibson \& M. Tallerman (Éds.), The Oxford Handbook of Language Evolution (p. 612-620). Oxford University Press.

de Vanssay-Maigne, A., Noulhiane, M., Devauchelle, A. D., Rodrigo, S., Baudoin-Chial, S., Meder, J. F., Oppenheim, C., Chiron, C., \& Chassoux, F. (2011). Modulation of encoding and retrieval by recollection and familiarity: Mapping the medial temporal lobe networks. NeuroImage, 58(4), 1131-1138. https://doi.org/10.1016/j.neuroimage.2011.06.086 
Diana, R. A., Yonelinas, A. P., \& Ranganath, C. (2007). Imaging recollection and familiarity in the medial temporal lobe: A three-component model. Trends in Cognitive Sciences, 11(9), 379-386. https://doi.org/10.1016/j.tics.2007.08.001

DiNicola, L. M., Braga, R. M., \& Buckner, R. L. (2020). Parallel Distributed Networks Dissociate Episodic and Social Functions Within the Individual. Journal of Neurophysiology, 123(3), 1144-1179.

Dohmatob, E., Dumas, G., \& Bzdok, D. (2020). Dark Control: The Default Mode Network as a Reinforcement Learning Agent. Human Brain Mapping, 38.

Doucet, G. E., Lee, W. H., \& Frangou, S. (2019). Evaluation of the spatial variability in the major resting-state networks across human brain functional atlases. Hum Brain Mapp, 4O(15), 4577-4587. https://doi.org/10.1002/hbm. 24722

Duff, M. C., \& Brown-Schmidt, S. (2012). The hippocampus and the flexible use and processing of language. Frontiers in Human Neuroscience, 6. https://doi.org/10.3389/fnhum.2012.00069

Duff, M. C., Covington, N. V., Hilverman, C., \& Cohen, N. J. (2020). Semantic Memory and the Hippocampus : Revisiting, Reaffirming, and Extending the Reach of Their Critical Relationship. Frontiers in Human Neuroscience, 13. https://doi.org/10.3389/fnhum.2019.00471

Duff, M. C., Hengst, J. A., Tranel, D., \& Cohen, N. J. (2009). Hippocampal amnesia disrupts verbal play and the creative use of language in social interaction. Aphasiology, 23(7-8), 926-939. https://doi.org/10.1080/02687030802533748

Duffau, H., Moritz-Gasser, S., \& Mandonnet, E. (2014). A re-examination of neural basis of language processing: Proposal of a dynamic hodotopical model from data provided by brain stimulation mapping during picture naming. Brain and Language, 131, 1-10. https://doi.org/10.1016/j.bandl.2013.05.011

Duvernoy, H. M., Cattin, F., \& Risold, P.-Y. (2013). The Human Hippocampus. Springer, Berlin Heidelberg. http://link.springer.com/10.1007/978-3-642-33603-4

Eisenberg, I. W., Bissett, P. G., Zeynep Enkavi, A., Li, J., MacKinnon, D. P., Marsch, L. A., \& Poldrack, R. A. (2019). Uncovering the structure of self-regulation through data-driven ontology discovery. Nature Communications, 10(1), 2319. https://doi.org/10.1038/s41467-019-10301-1

Ekstrom, A. D., \& Yonelinas, A. P. (2020). Precision, binding, and the hippocampus: Precisely what $\begin{array}{lllll}\text { are } \quad \text { talking } & & \end{array}$ https://doi.org/10.1016/j.neuropsychologia.2020.107341

Elverman, K. H., Resch, Z. J., Quasney, E. E., Sabsevitz, D. S., Binder, J. R., \& Swanson, S. J. (2019). Temporal lobe epilepsy is associated with distinct cognitive phenotypes. Epilepsy \& Behavior, 96, 61-68. https://doi.org/10.1016/j.yebeh.2019.04.015

Epstein, R. A. (2008). Parahippocampal and retrosplenial contributions to human spatial navigation. Trends in Cognitive Sciences, 12(10), 388-396. https://doi.org/10.1016/j.tics.2008.07.004

Eriksson, J., Vogel, E. K., Lansner, A., Bergström, F., \& Nyberg, L. (2015). Neurocognitive Architecture of Working Memory. Neuron, 33-46.

Everts, R., Simon Harvey, A., Lillywhite, L., Wrennall, J., Abbott, D. F., Gonzalez, L., Kean, M., Jackson, G. D., \& Anderson, V. (2010). Language lateralization correlates with verbal memory performance in children with focal epilepsy. Epilepsia, 51(4), 627-638. https://doi.org/10.1111/j.1528-1167.2009.02406.x 
Fabre-Thorpe, M. (2003). Visual categorization: Accessing abstraction in non-human primates. Phil. Trans. R. Soc. Lond. B, 358(1435), 1215-1223. https://doi.org/10.1098/rstb.2003.1310

Faskowitz, J., Esfahlani, F. Z., Jo, Y., Sporns, O., \& Betzel, R. F. (2020). Edge-centric functional network representations of human cerebral cortex reveal overlapping system-level architecture. Nature Neuroscience, 23(12), 1644-1654. https://doi.org/10.1038/s41593-020-00719-y

Ferreira, F., \& Engelhardt, P. E. (2006). Chapter 3-Syntax and Production. In M. J. Traxler \& M. A. Gernsbacher (Éds.), Handbook of Psycholinguistics (Second Edition) (p. 61-91). Academic Press. https://doi.org/10.1016/B978-012369374-7/50004-3

ffytche, D. H., \& Catani, M. (2005). Beyond localization: From hodology to function. Philosophical Transactions of the Royal Society B: Biological Sciences, 360(1456), 767-779. https://doi.org/10.1098/rstb.2005.1621

Fitch, W. T. (2010). The Evolution of Language. Cambridge University Press.

Forkel, S., Friedrich, P., Thiebaut de Schotten, M., \& Howells, H. (2020). White matter variability, cognition, and disorders: A systematic review. medRxiv.

Fornito, A., Zalesky, A., \& Breakspear, M. (2015). The connectomics of brain disorders. Nature Reviews. Neuroscience, 16(3), 159-172. https://doi.org/10.1038/nrn3901

Forseth, K., Pitkow, X., Fischer-Baum, S., \& Tandon, N. (2021). What The Brain Does As We Speak [Preprint]. Neuroscience. https://doi.org/10.1101/2021.02.05.429841

Friederici, A. D., Chomsky, N., Berwick, R. C., Moro, A., \& Bolhuis, J. J. (2017). Language, mind and brain. Nature Human Behaviour, 1(10), 713-722. https://doi.org/10.1038/s41562-017-0184-4

Friston, K. J., \& Price, C. J. (2003). Degeneracy and redundancy in cognitive anatomy. Trends in Cognitive Sciences, 74), 151-152. https://doi.org/10.1016/S1364-6613(03)00054-8

Gadian, D. G., Aicardi, J., Watkins, K. E., Porter, D. A., Mishkin, M., \& Vargha-Khadem, F. (2000). Developmental amnesia associated with early hypoxic-ischaemic injury. Brain, 123(3), 499-507. https://doi.org/10.1093/brain/123.3.499

Gaffan, D. (1997). Episodic and semantic memory and the role of the not-hippocampus. Trends in Cognitive Sciences, 1(7), 246-248. https://doi.org/10.1016/S1364-6613(97)01074-7

Geib, B. R., Stanley, M. L., Wing, E. A., Laurienti, P. J., \& Cabeza, R. (2017). Hippocampal Contributions to the Large-Scale Episodic Memory Network Predict Vivid Visual Memories. Cerebral Cortex, 27(1), 680-693. https://doi.org/10.1093/cercor/bhv272

Genon, S., Reid, A., Langner, R., Amunts, K., \& Eickhoff, S. B. (2018). How to Characterize the Function of a Brain Region. Trends in Cognitive Sciences, 22(4), 350-364. https://doi.org/10.1016/j.tics.2018.01.010

Giraud, A.-L., \& Poeppel, D. (2012). Cortical oscillations and speech processing: Emerging computational principles and operations. Nature neuroscience, 15(4), 511-517. https://doi.org/10.1038/nn.3063

Goldstein, A., Zada, Z., Buchnik, E., Schain, M., Price, A., Aubrey, B., Nastase, S. A., Feder, A., Emanuel, D., Cohen, A., Jansen, A., Gazula, H., Choe, G., Rao, A., Kim, C., Casto, C., Fanda, L., Doyle, W., Friedman, D., ... Hasson, U. (2021). Thinking ahead: Spontaneous prediction in context as a keystone of language in humans and machines (p. 2020.12.02.403477). https://doi.org/10.1101/2020.12.02.403477 
Gordon, E. M., Laumann, T. O., Marek, S., Raut, R. V., Gratton, C., Newbold, D. J., Greene, D. J., Coalson, R. S., Snyder, A. Z., Schlaggar, B. L., Petersen, S. E., Dosenbach, N. U. F., \& Nelson, S. M. (2020). Default-mode network streams for coupling to language and control systems. Proceedings of the National Academy of Sciences, 117(29), 17308-17319. https://doi.org/10.1073/pnas.2005238117

Goucha, T., Zaccarella, E., \& Friederici, A. D. (2017). A revival of Homo loquens as a builder of labeled structures: Neurocognitive considerations. Neuroscience \& Biobehavioral Reviews, 81, 213-224. https://doi.org/10.1016/j.neubiorev.2017.01.036

Graham, K. S., Simons, J. S., Pratt, K. H., Patterson, K., \& Hodges, J. R. (2000). Insights from semantic dementia on the relationship between episodic and semantic memory. Neuropsychologia, 38(3), 313-324. https://doi.org/10.1016/S0028-3932(99)00073-1

Gratton, C., Nomura, E. M., Pérez, F., \& D'Esposito, M. (2012). Focal Brain Lesions to Critical Locations Cause Widespread Disruption of the Modular Organization of the Brain. Journal of Cognitive Neuroscience, 24(6), 1275-1285. https://doi.org/10.1162/jocn_a_00222

Guell, X., \& Schmahmann, J. (2020). Cerebellar Functional Anatomy: A Didactic Summary Based on Human fMRI Evidence. Cerebellum, 19(1), 1-5. https://doi.org/10.1007/s12311-019-01083-9

Hagoort, P. (2013). MUC (Memory, Unification, Control) and beyond. Frontiers in Psychology, 4, 416. https://doi.org/10.3389/fpsyg.2013.00416

Hagoort, P. (2014). Nodes and networks in the neural architecture for language: Broca's region and beyond. Current Opinion in Neurobiology, $136-141$. https://doi.org/10.1016/j.conb.2014.07.013

Hagoort, P. (2016). MUC (Memory, Unification, Control): A Model on the Neurobiology of Language Beyond Single Word Processing. In G. Hickok \& S. L. Small (Éds.), Neurobiology of Language (p. 339-347). Elsevier. https://doi.org/10.1016/B978-0-12-407794-2.00028-6

Hagoort, P. (2019). The neurobiology of language beyond single-word processing. Science. https://www.science.org/doi/abs/10.1126/science.aax0289

Hamamé, C. M., Alario, F.-X., Llorens, A., Liégeois-Chauvel, C., \& Trébuchon-Da Fonseca, A. (2014). High frequency gamma activity in the left hippocampus predicts visual object naming performance. Brain and Language, 135, 104-114. https://doi.org/10.1016/j.bandl.2014.05.007

Hasson, U., Chen, J., \& Honey, C. J. (2015). Hierarchical process memory: Memory as an integral component of information processing. Trends in Cognitive Sciences, 19(6), 304-313. https://doi.org/10.1016/j.tics.2015.04.006

Herbet, G., \& Duffau, H. (2020). Revisiting the Functional Anatomy of the Human Brain: Toward a Meta-Networking Theory of Cerebral Functions. Physiological Reviews. https://doi.org/10.1152/physrev.00033.2019

Hickok, G., \& Poeppel, D. (2007). The cortical organization of speech processing. Nature Reviews Neuroscience, 8(5), 393-402. https://doi.org/10.1038/nrn2113

Higgins, C., Liu, Y., Vidaurre, D., Kurth-Nelson, Z., Dolan, R., Behrens, T., \& Woolrich, M. (2020). Replay bursts coincide with activation of the default mode and parietal alpha network. BioRxiv, 2020.06.23.166645. https://doi.org/10.1101/2020.06.23.166645

Hilgetag, C. C., \& Goulas, A. (2020). 'Hierarchy' in the organization of brain networks. Philosophical Transactions of the Royal Society B: Biological Sciences, 375(1796), 20190319. https://doi.org/10.1098/rstb.2019.0319 
Hilverman, C., \& Duff, M. C. (2021). Evidence of impaired naming in patients with hippocampal amnesia. Hippocampus, 31(6), 612-626. https://doi.org/10.1002/hipo.23325

Hinzen, W. (2013). Narrow syntax and the language of thought. Philosophical Psychology, 26(1), 1-23. https://doi.org/10.1080/09515089.2011.627537

Hogan, T. P., Adlof, S. M., \& Alonzo, C. N. (2014). On the importance of listening comprehension. International Journal of Speech-Language Pathology, 16(3), 199-207. https://doi.org/10.3109/17549507.2014.904441

Horton, W. S. (2007). The influence of partner-specific memory associations on language production: Evidence from picture naming. Language and Cognitive Processes, 22(7), 1114-1139. https://doi.org/10.1080/01690960701402933

Hula, W. D., Panesar, S., Gravier, M. L., Yeh, F.-C., Dresang, H. C., Dickey, M. W., \& FernandezMiranda, J. C. (2020). Structural white matter connectometry of word production in aphasia: An observational study. Brain, 143(8), 2532-2544. https://doi.org/10.1093/brain/awaa193

Humphreys, G. F., \& Lambon Ralph, M. A. (2015). Fusion and Fission of Cognitive Functions in the Human Parietal Cortex. Cereb. Cortex, 25(10), 3547-3560. https://doi.org/10.1093/cercor/bhu198

Indefrey, P. (2011). The Spatial and Temporal Signatures of Word Production Components : A Critical Update. Frontiers in Psychology, 2. https://doi.org/10.3389/fpsyg.2011.00255

Jaimes-Bautista, A. G., Rodríguez-Camacho, M., Martínez-Juárez, I. E., \& Rodríguez-Agudelo, Y. (2015, juillet 16). Semantic Processing Impairment in Patients with Temporal Lobe Epilepsy [Review Article]. Epilepsy Research and Treatment; Hindawi. https://doi.org/10.1155/2015/746745

Jain, S., \& Huth, A. (2018). Incorporating Context into Language Encoding Models for fMRI. Advances in Neural Information Processing 31. https://papers.nips.cc/paper/2018/hash/f471223d1a1614b58a7dc45c9d01df19-Abstract.html

Jeong, W., Chung, C. K., \& Kim, J. S. (2015). Episodic memory in aspects of large-scale brain networks. Front. Hum. Neurosci., 9. https://doi.org/10.3389/fnhum.2015.00454

Ji, J. L., Spronk, M., Kulkarni, K., Repovš, G., Anticevic, A., \& Cole, M. W. (2019, 15). Mapping the human brain's cortical-subcortical functional network organization. NeuroImage, 35-57.

Johnson, E. L., \& Knight, R. T. (2015). Intracranial recordings and human memory. Current Opinion in Neurobiology, 31, 18-25. https://doi.org/10.1016/j.conb.2014.07.021

Jonin, P.-Y., Calia, C., Muratot, S., Belliard, S., Duché, Q., Barbeau, E. J., \& Parra, M. A. (2019). Refining understanding of working memory buffers through the construct of binding: Evidence from a single case informs theory and clinical practise. Cortex; a Journal Devoted to the Study of the Nervous System and Behavior, 112, 37-57. https://doi.org/10.1016/j.cortex.2018.08.011

Kempe, V., \& Brooks, P. J. (2016). Modern Theories of Language. In V. Weekes-Shackelford, T. K. Shackelford, \& V. A. Weekes-Shackelford (Éds.), Encyclopedia of Evolutionary Psychological Science (p. 1-12). Springer International Publishing. http://link.springer.com/10.1007/978-3-31916999-6_3321-1

Kikuchi, H., Fujii, T., Abe, N., Suzuki, M., Takagi, M., Mugikura, S., Takahashi, S., \& Mori, E. (2010). Memory Repression: Brain Mechanisms underlying Dissociative Amnesia. Journal of Cognitive Neuroscience, 22(3), 602-613. https://doi.org/10.1162/jocn.2009.21212 
Kintsch, W., \& Kintsch, E. (2005). Comprehension. In S. G. Paris \& S. A. Stahl (Éds.), Children's reading comprehension and assessment (p. 71-92). Routledge.

Klemfuss, J. Z. (2015). Differential Contributions of Language Skills to Children's Episodic Recall. Journal of Cognition and Development, 16(4), 608-620. https://doi.org/10.1080/15248372.2014.952415

Kliemann, D., Adolphs, R., Tyszka, J. M., Fischl, B., Yeo, B. T. T., Nair, R., Dubois, J., \& Paul, L. K. (2019). Intrinsic Functional Connectivity of the Brain in Adults with a Single Cerebral Hemisphere. Cell Reports, 29(8), 2398-2407.e4. https://doi.org/10.1016/j.celrep.2019.10.067

Konishi, M., McLaren, D. G., Engen, H., \& Smallwood, J. (2015). Shaped by the Past: The Default Mode Network Supports Cognition that Is Independent of Immediate Perceptual Input. PLOS ONE, 10(6), e0132209. https://doi.org/10.1371/journal.pone.0132209

Krajcovicova, L., Marecek, R., Mikl, M., \& Rektorova, I. (2014). Disruption of Resting Functional Connectivity in Alzheimer's Patients and At-Risk Subjects. Current Neurology and Neuroscience Reports, 14(10), 491. https://doi.org/10.1007/s11910-014-0491-3

Krienen, F. M., Tu, P.-C., \& Buckner, R. L. (2010). Clan Mentality: Evidence That the Medial Prefrontal Cortex Responds to Close Others. Journal of Neuroscience, 30(41), 13906-13915. https://doi.org/10.1523/JNEUROSCI.2180-10.2010

Larsen, S. F., Schrauf, R. W., Fromholt, P., \& Rubin, D. C. (2002). Inner speech and bilingual autobiographical memory: A Polish-Danish cross-cultural study. Memory, 10(1), 45-54. https://doi.org/10.1080/09658210143000218

Lau, E. F., Gramfort, A., Hämäläinen, M. S., \& Kuperberg, G. R. (2013). Automatic Semantic Facilitation in Anterior Temporal Cortex Revealed through Multimodal Neuroimaging. $J$. Neurosci., 33(43), 17174-17181. https://doi.org/10.1523/JNEUROSCI.1018-13.2013

Levelt, W. J. M. (1989). Speaking: From intention to articulation. MIT Press.

Levelt, W. J. M. (1993). Lexical Access in Speech Production. In E. Reuland \& W. Abraham (Éds.), Knowledge and Language: Volume I From Orwell's Problem to Plato's Problem (p. 241-251). Springer Netherlands. https://doi.org/10.1007/978-94-011-1840-8_11

Leventhal, D. K., Gage, G. J., Schmidt, R., Pettibone, J. R., Case, A. C., \& Berke, J. D. (2012). Basal Ganglia Beta Oscillations Accompany Cue Utilization. Neuron, 73(3), 523-536. https://doi.org/10.1016/j.neuron.2011.11.032

Libby, L. A., Hannula, D. E., \& Ranganath, C. (2014). Medial Temporal Lobe Coding of Item and Spatial Information during Relational Binding in Working Memory. Journal of Neuroscience, 34(43), 14233-14242. https://doi.org/10.1523/JNEUROSCI.0655-14.2014

Luo, C., Makaretz, S., Stepanovic, M., Papadimitriou, G., Quimby, M., Palanivelu, S., Dickerson, B. C., \& Makris, N. (2020). Middle longitudinal fascicle is associated with semantic processing deficits in primary progressive aphasia. NeuroImage: Clinical, 25, 102115. https://doi.org/10.1016/j.nicl.2019.102115

Lynn, C. W., \& Bassett, D. S. (2019). The physics of brain network structure, function and control. Nature Reviews Physics, 1(5), 318-332. https://doi.org/10.1038/s42254-019-0040-8

Margulies, D. S., Ghosh, S. S., Goulas, A., Falkiewicz, M., Huntenburg, J. M., Langs, G., Bezgin, G., Eickhoff, S. B., Castellanos, F. X., Petrides, M., Jefferies, E., \& Smallwood, J. (2016). Situating the default-mode network along a principal gradient of macroscale cortical organization. 
Proceedings of the National Academy of Sciences, 113(44), 12574-12579. https://doi.org/10.1073/pnas.1608282113

Marian, V., \& Neisser, U. (2000). Language-Dependent Recall of Autobiographical Memories. Journal of Experimental Psychology: General, 126(3), 361-368.

Marr, D. (1982). Vision: A computational investigation into the human representation and processing of visual information.

Martin, A. E. (2020). A Compositional Neural Architecture for Language. Journal of Cognitive Neuroscience, 32(8), 1407-1427. https://doi.org/10.1162/jocn_a_01552

Matthen, M. (2005). Seeing, Doing, and Knowing: A Philosophical Theory of Sense Perception. Oxford University Press.

Mazoyer, B., Zago, L., Mellet, E., Bricogne, S., Etard, O., Houdé, O., Crivello, F., Joliot, M., Petit, L., \& Tzourio-Mazoyer, N. (2001). Cortical networks for working memory and executive functions sustain the conscious resting state in man. Brain Research Bulletin, 54(3), 287-298. https://doi.org/10.1016/S0361-9230(00)00437-8

McCormick, C., Protzner, A. B., Barnett, A. J., Cohn, M., Valiante, T. A., \& McAndrews, M. P. (2014). Linking DMN connectivity to episodic memory capacity: What can we learn from patients with medial temporal lobe damage? Neurolmage: Clinical, 5, 188-196. https://doi.org/10.1016/j.nicl.2014.05.008

Medaglia, J. D., \& Bassett, D. S. (2018, septembre 26). Network Analyses and Nervous System Disorders. Oxford Research Encyclopedia of https://doi.org/10.1093/acrefore/9780190264086.013.121

Mesulam, M.-M. (1990). Large-scale neurocognitive networks and distributed processing for attention, language, and memory. Annals of Neurology, 28(5), 597-613. https://doi.org/10.1002/ana.410280502

Metternich, B., Buschmann, F., Wagner, K., Schulze-Bonhage, A., \& Kriston, L. (2014). Verbal fluency in focal epilepsy: A systematic review and meta-analysis. Neuropsychology Review, 24(2), 200-218. https://doi.org/10.1007/s11065-014-9255-8

Milner, B. (1970). Memory and the medial temporal regions of the brain. Biology of Memory, 23, 31-59.

Moscovitch, M., Cabeza, R., Winocur, G., \& Nadel, L. (2016). Episodic Memory and Beyond: The Hippocampus and Neocortex in Transformation. Annu. Rev. Psychol., 67(1), 105-134. https://doi.org/10.1146/annurev-psych-113011-143733

Moscovitch, M., Nadel, L., Winocur, G., Gilboa, A., \& Rosenbaum, R. S. (2006). The cognitive neuroscience of remote episodic, semantic and spatial memory. Current Opinion in Neurobiology, 16(2), 179-190. https://doi.org/10.1016/j.conb.2006.03.013

Murphy, E. (2015a). The brain dynamics of linguistic computation. Frontiers in Psychology, 6, 1515. https://doi.org/10.3389/fpsyg.2015.01515

Murphy, E. (2015b). Labels, cognomes, and cyclic computation: An ethological perspective. Front. Psychol., 6. https://doi.org/10.3389/fpsyg.2015.00715

Noppeney, U., Friston, K. J., \& Price, C. J. (2004). Degenerate neuronal systems sustaining cognitive functions. Journal of Anatomy, 205(6), 433-442. https://doi.org/10.1111/j.0021-8782.2004.00343.x 
Oulhaj, A., Wilcock, G. K., Smith, A. D., \& de Jager, C. A. (2009). Predicting the time of conversion to MCI in the elderly: Role of verbal expression and learning. Neurology, 73(18), 1436-1442. https://doi.org/10.1212/WNL.0b013e3181c0665f

Ovadia-Caro, S., Villringer, K., Fiebach, J., Jungehulsing, G. J., van der Meer, E., Margulies, D. S., \& Villringer, A. (2013). Longitudinal Effects of Lesions on Functional Networks after Stroke. J Cereb Blood Flow Metab, 33(8), 1279-1285. https://doi.org/10.1038/jcbfm.2013.80

Palacio, N., \& Cardenas, F. (2019). A systematic review of brain functional connectivity patterns involved in episodic and semantic memory. Reviews in the Neurosciences, 30(8), 889-902. https://doi.org/10.1515/revneuro-2018-0117

Parr, T., \& Friston, K. J. (2017). Working memory, attention, and salience in active inference. Sci Rep, 71), 14678. https://doi.org/10.1038/s41598-017-15249-0

Piai, V., Anderson, K. L., Lin, J. J., Dewar, C., Parvizi, J., Dronkers, N. F., \& Knight, R. T. (2016). Direct brain recordings reveal hippocampal rhythm underpinnings of language processing. Proceedings of the National Academy of Sciences, 113(40), 11366-11371. https://doi.org/10.1073/pnas.1603312113

Pidgeon, L. M., \& Morcom, A. M. (2016). Cortical pattern separation and item-specific memory encoding. Neuropsychologia, 85, 256-271. https://doi.org/10.1016/j.neuropsychologia.2016.03.026

Poeppel, D., \& Assaneo, M. F. (2020). Speech rhythms and their neural foundations. Nature Reviews Neuroscience, 21(6), 322-334. https://doi.org/10.1038/s41583-020-0304-4

Power, J. D., Schlaggar, B. L., Lessov-Schlaggar, C. N., \& Petersen, S. E. (2013). Evidence for hubs in human functional brain networks. Neuron, 79(4), 10.1016/j.neuron.2013.07.035. https://doi.org/10.1016/j.neuron.2013.07.035

Premack, D., \& Woodruff, G. (1978). Does the chimpanzee have a theory of mind? Behavioral and brain sciences, 1(4), 515-526.

Price, C. J. (2012). A review and synthesis of the first 20years of PET and fMRI studies of heard speech, $\begin{array}{llll}\text { spoken language and } & \text { reading. }\end{array}$ https://doi.org/10.1016/j.neuroimage.2012.04.062

Raichle, M. E. (2015). The Brain's Default Mode Network. Annual Review of Neuroscience, 38(1), 433-447. https://doi.org/10.1146/annurev-neuro-071013-014030

Raichle, M. E., MacLeod, A. M., Snyder, A. Z., Powers, W. J., Gusnard, D. A., \& Shulman, G. L. (2001). A default mode of brain function. Proceedings of the National Academy of Sciences, 98(2), 676-682. https://doi.org/10.1073/pnas.98.2.676

Ranganath, C., \& Ritchey, M. (2012). Two cortical systems for memory-guided behaviour. Nature Reviews Neuroscience, 13(10), 713-726. https://doi.org/10.1038/nrn3338

Rangel, L. M., Rueckemann, J. W., Riviere, P. D., Keefe, K. R., Porter, B. S., Heimbuch, I. S., Budlong, C. H., \& Eichenbaum, H. (2016). Rhythmic coordination of hippocampal neurons during associative memory processing. ELife, 5, e09849. https://doi.org/10.7554/eLife.09849

Reuland, E. (2017). Language and imagination: Evolutionary explorations. Neuroscience \& Biobehavioral Reviews, 81, 255-278. https://doi.org/10.1016/j.neubiorev.2016.12.017

Reyes, A., Kaestner, E., Ferguson, L., Jones, J. E., Seidenberg, M., Barr, W. B., Busch, R. M., Hermann, B. P., \& McDonald, C. R. (2020). Cognitive phenotypes in temporal lobe epilepsy utilizing dataand clinically driven approaches: Moving toward a new taxonomy. Epilepsia, 1211-1220. 
Richter, F. R., Cooper, R. A., Bays, P. M., \& Simons, J. S. (2016). Distinct neural mechanisms underlie the success, precision, and vividness of episodic memory. ELife, 5, e18260. https://doi.org/10.7554/eLife.18260

Rilling, J. K. (2014). Comparative primate neuroimaging: Insights into human brain evolution. Trends in Cognitive Sciences, 18(1), 46-55. https://doi.org/10.1016/j.tics.2013.09.013

Ritchey, M., Montchal, M. E., Yonelinas, A. P., \& Ranganath, C. (2015). Delay-dependent contributions of medial temporal lobe regions to episodic memory retrieval. eLife, 4, e05025. https://doi.org/10.7554/eLife.05025

Roger, E. (2020). Neurocognitive reorganizations of language and memory in temporal lobe epilepsy: A multimodal connectivity-based approach [Doctoral thesis, Grenoble Alpes University]. http://www.theses.fr/2020GRALS011

Roger, E., Pichat, C., Torlay, L., David, O., Renard, F., Banjac, S., Attyé, A., Minotti, L., Lamalle, L., Kahane, P., \& Baciu, M. (2019). Hubs disruption in mesial temporal lobe epilepsy. A resting-state fMRI study on a language-and-memory network. Human Brain Mapping, 41(3), 779-796. https://doi.org/10.1002/hbm.24839

Roger, E., Torlay, L., Gardette, J., Mosca, C., Banjac, S., Minotti, L., Kahane, P., \& Baciu, M. (2020). A machine learning approach to explore cognitive signatures in patients with temporo-mesial epilepsy. Neuropsychologia, 142, 107455. https://doi.org/10.1016/j.neuropsychologia.2020.107455

Rosazza, C., \& Minati, L. (2011). Resting-state brain networks: Literature review and clinical applications. Neurological Sciences, 32(5), 773-785. https://doi.org/10.1007/s10072-011-0636-y

Santi, A., Friederici, A. D., Makuuchi, M., \& Grodzinsky, Y. (2015). An fMRI study dissociating distance measures computed by Broca's area in movement processing: Clause boundary vs. identity. Front. Psychol., 6, 654. https://doi.org/10.3389/fpsyg.2015.00654

Schaeffer, J. D. (1990). Sensus Communis : Vico, Rhetoric, and the Limits of Relativism. Duke University Press.

Schwering, S. C., \& MacDonald, M. C. (2020). Verbal Working Memory as Emergent from Language Comprehension and Production. Frontiers in Human Neuroscience, 14, 68. https://doi.org/10.3389/fnhum.2020.00068

Scoville, W. B., \& Milner, B. (1957). Loss Of Recent Memory After Bilateral Hippocampal Lesions. Journal of Neurology, Neurosurgery, and Psychiatry, 20(1), 11-21.

Sheldon, S., Farb, N., Palombo, D. J., \& Levine, B. (2016). Intrinsic medial temporal lobe connectivity relates to individual differences in episodic autobiographical remembering. Cortex, 74, 206-216. https://doi.org/10.1016/j.cortex.2015.11.005

Siegel, J. S., Ramsey, L. E., Snyder, A. Z., Metcalf, N. V., Chacko, R. V., Weinberger, K., Baldassarre, A., Hacker, C. D., Shulman, G. L., \& Corbetta, M. (2016). Disruptions of network connectivity predict impairment in multiple behavioral domains after stroke. Proc Natl Acad Sci USA, 113(30), E4367-E4376. https://doi.org/10.1073/pnas.1521083113

Silbert, L. J., Honey, C. J., Simony, E., Poeppel, D., \& Hasson, U. (2014). Coupled neural systems underlie the production and comprehension of naturalistic narrative speech. Proceedings of the National Academy of Sciences, 111(43), E4687-E4696. https://doi.org/10.1073/pnas.1323812111

Simony, E., Honey, C. J., Chen, J., Lositsky, O., Yeshurun, Y., Wiesel, A., \& Hasson, U. (2016). Dynamic reconfiguration of the default mode network during narrative comprehension. Nat Commun, 7(1), 12141. https://doi.org/10.1038/ncomms12141 
Sneve, M. H., Grydeland, H., Amlien, I. K., Langnes, E., Walhovd, K. B., \& Fjell, A. M. (2017). Decoupling of large-scale brain networks supports the consolidation of durable episodic memories. NeuroImage, 153, 336-345. https://doi.org/10.1016/j.neuroimage.2016.05.048

Spiers, H. J. (2020). The Hippocampal Cognitive Map: One Space or Many? Trends in Cognitive Sciences, S1364661319303158. https://doi.org/10.1016/j.tics.2019.12.013

Spreng, R. N., Sepulcre, J., Turner, G. R., Stevens, W. D., \& Schacter, D. L. (2013). Intrinsic Architecture Underlying the Relations among the Default, Dorsal Attention, and Frontoparietal Control Networks of the Human Brain. Journal of Cognitive Neuroscience, 25(1), 74-86. https://doi.org/10.1162/jocn_a_00281

Squire, L. R. (2004). Memory systems of the brain : A brief history and current perspective. Neurobiology of Learning and Memory, 82(3), 171-177. https://doi.org/10.1016/j.nlm.2004.06.005

Squire, L. R. (2009). The Legacy of Patient H.M. for Neuroscience. Neuron, 61(1), 6-9. https://doi.org/10.1016/j.neuron.2008.12.023

Squire, S., L. R. (1984). The Neuropsychology of Memory. In P. Marler \& H. S. Terrace (Éds.), The Biology of Learning. Dahlem Workshop Reports (Life Sciences Research Reports) (Vol. 29, p. 667-686). Springer.

Sreekumar, V., Nielson, D. M., Smith, T. A., Dennis, S. J., \& Sederberg, P. B. (2018). The experience of vivid autobiographical reminiscence is supported by subjective content representations in the precuneus. Sci Rep, 8(1), 14899. https://doi.org/10.1038/s41598-018-32879-0

Studdert-Kennedy, M. (2005). How did language go discrete. In M. Tallerman (Éd.), Language origins: Perspectives on evolution (p. 48-67). Oxford University Press.

Thiebaut de Schotten, M., Dell'Acqua, F., Ratiu, P., Leslie, A., Howells, H., Cabanis, E., Iba-Zizen, M. T., Plaisant, O., Simmons, A., Dronkers, N. F., Corkin, S., \& Catani, M. (2015). From Phineas Gage and Monsieur Leborgne to H.M.: Revisiting Disconnection Syndromes. Cerebral Cortex (New York, NY), 25(12), 4812-4827. https://doi.org/10.1093/cercor/bhv173

Tulving, E. (1972). Episodic and semantic memory. In Organization of memory. (p. xiii, 423-xiii, 423). Academic Press.

Tyszka, J. M., Kennedy, D. P., Adolphs, R., \& Paul, L. K. (2011). Intact Bilateral Resting-State Networks in the Absence of the Corpus Callosum. Journal of Neuroscience, 31(42), 15154-15162. https://doi.org/10.1523/JNEUROSCI.1453-11.2011

Uddin, L. Q. (2020). An 'edgy' new look. Nature Neuroscience, 23(12), 1471-1472. https://doi.org/10.1038/s41593-020-00741-0

Vargha-Khadem, F., \& Cacucci, F. (2021). A brief history of developmental amnesia. Neuropsychologia, 150, 107689. https://doi.org/10.1016/j.neuropsychologia.2020.107689

Vargha-Khadem, F., Gadian, D. G., Watkins, K. E., Connelly, A., Paesschen, W. V., \& Mishkin, M. (1997). Differential Effects of Early Hippocampal Pathology on Episodic and Semantic Memory. Science. https://www.science.org/doi/abs/10.1126/science.277.5324.376

Walenski, M., Europa, E., Caplan, D., \& Thompson, C. K. (2019). Neural networks for sentence comprehension and production: An ALE-based meta-analysis of neuroimaging studies. Human Brain Mapping, 4O(8), 2275-2304. https://doi.org/10.1002/hbm.24523 
Wang, S., Van der Haegen, L., Tao, L., \& Cai, Q. (2019). Brain Functional Organization Associated With Language Lateralization. Cerebral Cortex, 29(10), 4312-4320. https://doi.org/10.1093/cercor/bhy313

Wang, X., Margulies, D. S., Smallwood, J., \& Jefferies, E. (2020). A gradient from long-term memory to novel cognition: Transitions through default mode and executive cortex [Preprint]. Neuroscience. https://doi.org/10.1101/2020.01.16.908327

Waxman, S. R. (2004). Everything had a name, and each name gave birth to a new thought: Links between early word-learning and conceptual organization. In G. D. Hall \& S. R. Waxman (Éds.), Weaving a lexicon (p. 295-335).

Wernicke, C. (1874). Der aphasische Symptomencomplex: Eine psychologische Studie auf anatomischer Basis. Cohn.

Wilson, S. M., Galantucci, S., Tartaglia, M. C., \& Gorno-Tempini, M. L. (2012). The neural basis of syntactic deficits in primary progressive aphasia. Brain and Language, 122(3), 190-198. https://doi.org/10.1016/j.bandl.2012.04.005

Wolff, M., \& Vann, S. D. (2019). The Cognitive Thalamus as a Gateway to Mental Representations. J. Neurosci., 39(1), 3-14. https://doi.org/10.1523/JNEUROSCI.0479-18.2018

Xu, Y., He, Y., \& Bi, Y. (2017). A Tri-network Model of Human Semantic Processing. Frontiers in Psychology, 8. https://doi.org/10.3389/fpsyg.2017.01538

Yang, M., Logothetis, N. K., \& Eschenko, O. (2019). Occurrence of Hippocampal Ripples is Associated with Activity Suppression in the Mediodorsal Thalamic Nucleus. Journal of Neuroscience, 39(3), 434-444. https://doi.org/10.1523/JNEUROSCI.2107-18.2018

Yarkoni, T., Poldrack, R. A., Nichols, T. E., Van Essen, D. C., \& Wager, T. D. (2011). Large-scale automated synthesis of human functional neuroimaging data. Nature Methods, 8(8), 665-670. https://doi.org/10.1038/nmeth.1635

Yassa, M. A., \& Stark, C. E. L. (2011). Pattern separation in the hippocampus. Trends in Neurosciences, 34(10), 515-525. https://doi.org/10.1016/j.tins.2011.06.006

Yeo, T., Krienen, F. M., Sepulcre, J., Sabuncu, M. R., Lashkari, D., Hollinshead, M., Roffman, J. L., Smoller, J. W., Zöllei, L., Polimeni, J. R., Fischl, B., Liu, H., \& Buckner, R. L. (2011). The organization of the human cerebral cortex estimated by intrinsic functional connectivity. Journal of Neurophysiology, 106(3), 1125-1165. https://doi.org/10.1152/jn.00338.2011

Yonelinas, A. P., \& Ritchey, M. (2015). The slow forgetting of emotional episodic memories: An emotional binding account. Trends in Cognitive Sciences, 19(5), 259-267. https://doi.org/10.1016/j.tics.2015.02.009

Zaccarella, E., \& Friederici, A. D. (2015). Merge in the Human Brain: A Sub-Region Based Functional Investigation in the Left Pars Opercularis. Frontiers in Psychology, 6. https://doi.org/10.3389/fpsyg.2015.01818

Zhao, F., Kang, H., Llbo, Y., Rastogi, P., Venkatesh, D., \& Chandra, M. (2014). Neuropsychological deficits in temporal lobe epilepsy: A comprehensive review. Ann Indian Acad Neurol, 17(4), 374-382. https://doi.org/10.4103/0972-2327.144003 


\section{Box 1: Traditional "cognitive" definitions associated with language and memory}

Language is classically defined as a natural, intrinsic, and universal ability of human beings to construct communication systems using codes (speech sounds or written symbols) and to use these codes. Language cognitively involves a semantic system (vocabulary and lexical access), specialized sensory-motor capacities of perception and production (phonology), as well as capacities for decoding, manipulating (grammar/syntax), and understanding these codes (shared symbolism; comprehension).

Language production can be defined as the physical signal used to transmit language and share thoughts. Speech production would require, among other capacities, syntactic and articulation processes.

Syntax refers to the rules for organizing elements - word segments, words, sentences into a grammatical discourse - to generate combinatorial and hierarchical structures.

Verbal comprehension encompasses various processes helping to construct understandable and meaningful speech productions (expressive language skills up to the pragmatics of language, for instance) and to understand verbal productions (receptive language skills echoing vocabulary or semantics).

Memory represents the ability to maintain information or representations of past experience or knowledge, arguing to be based on mental processes of encoding, retention and retrieval, or reactivation. Several forms of memory have been proposed depending, for example, on the degree of consciousness or attention given to the process (implicit versus explicit memorization) and/or the duration of retention (short-term versus long-term memorization).

Working memory is positioned between short and long-term memory and concerns the ability to explicitly maintain and manipulate (re-)instantiated information to perform complex cognitive tasks of learning, reasoning or comprehension. Working memory is generally considered to be part of executive functioning (or central executive system), covering concepts such as planning, inhibition, and mental flexibility.

Verbal working memory involves a system for programming the utterance, scheduling verbal items at several levels (words, phonemes and articulatory gestures), and maintaining what needs to be produced (phonological loop and rehearsal); and appears conceptually close to the definition of syntax.

Declarative memory is involved in maintaining information about facts/knowledge or events for a significant period of time (long-term memory) and consciously recalling information. Declarative memory is classically divided into two subtypes: semantic memory and episodic memory.

Semantic memory includes general and factual knowledge about the world and abstract concepts (noetic consciousness). It allows individuals to make sense of information and/or to engage in cognitive processes such as object recognition or appropriate language use.

Episodic memory evokes the memory of personally experienced events associated with a particular time and place (spatiotemporal context), involving a sense of self-awareness (or autonoetic consciousness). In addition to the conscious recall of past events, episodic memory 
implies a "mental journey through time" (mental time travel, i.e., a projection into the past and/or future).

Associative memory: retrieval or activation of memories (stimulus, behaviors, facts, events...) conceptually or contextually associated.

Note: Definitions are extracted primarily from the dictionary of the American Psychological Association (https://dictionary.apa.org). Definitions may vary from one theorist to another, but it is interesting to note that there are overlaps and bridges between the different subfunctions described for language and declarative memory. These interconnections can be expressed as a cognitive network (Figure Box 1).

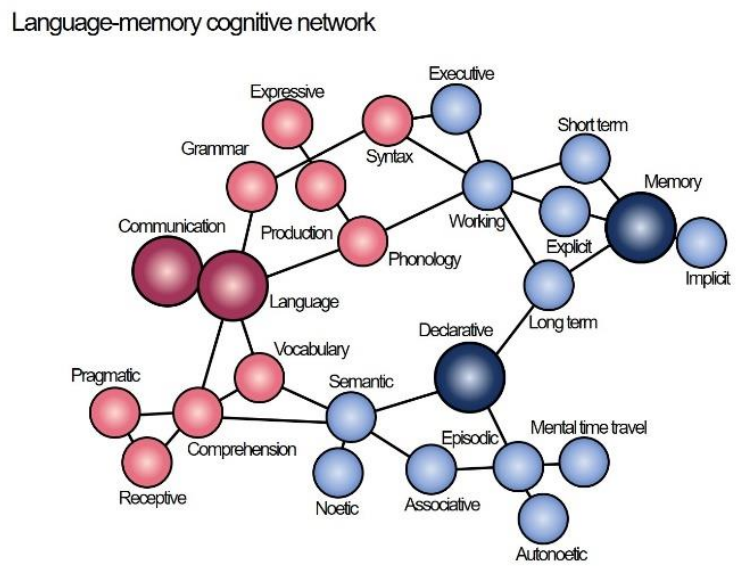




\section{Box 2: LUM's fundamentals and outstanding questions}

Brain mapping is traditionally delineated from psychological manifestations. This classical approach restricts the field of study to inherited and intangible constructs often speculative, ambiguous, and/or chimerical that compromise our understanding of neurocognitive functioning (blurred and unspecific cognitive labels; Buzsáki, 2020). The brain-behavior discrepancy is of particular concern for complex or high-level cognitive functions, such as language and memory. LUM (Language/union/Memory) faces the problem by considering language and memory in a unified way and as a single behavior. We formalize the unification of language and declarative memory by adopting a neuro-centric framework (as opposed to mainstream cognitive-centric approaches) based on brain connectivity findings.

The main tenets can be outlined as follows:

(i) Structure/Spatial: cognitive processes initially defined distinctively are entangled (Box 1) and share a mutual brain ground (see, e.g., Eisenberg et al., 2019). Three fundamental language-memory overlapping dimensions can be considered in the LUM frame (Figure 2). See also Section 2.3 and Figure 3 for a specification of the structures at the languagememory intersection.

(ii) Dynamics/Temporal: behavior emanates from the synergy of highly interactive functional brain dynamics (e.g., Cocchi et al., 2013; Cocuzza et al., 2020; Hagoort, 2019 and its multi-network perspective in language). LUM proposes a central architecture consisting of a triplet of main networks whose interactions can underlie the latent dimensions of the language-memory behavior (Section 3.2 and Figure 4).

(iii) Implementation/Processing: the spectrum of observable behaviors depends on an embedding of local (regional) and global (states) brain dynamics that support specialized operations (neuroperations; Cowell et al., 2019). Figure 5 describes an example of these nested global and local processes underpinning operations involved in the LUM context at the intersection of language and memory (see also Section 4.1).

(iv) Equilibrium/Plasticity: cognitive/behavioral efficiency is determined by states or states equilibrium that is modulated by idiosyncratic factors of variability (see Herbet \& Duffau, 2020, for instance). Section 4.2 describes several LUM disturbances in several brain affections. Figure 6 shows a concrete example in the case of chronic (epileptic) mesial temporal lobe damage.

The fundamental principles underlying LUM are not limited to the processing of verbal information and can be enriched in the future. This framework implies considering functional specialization or perturbation under a different frame of reference, moving away from traditional cognitive concepts.

Some important questions remain open and need to be addressed in the future:

- What investigative methods/tools and level of granularity are optimal for identifying elementary/primitive neuroperations?

- Can computational sciences help to draw a parallel with brain operations? In other words, is the language of the brain similar to that of (current) algorithms?

- Can we envisage a common understanding of brain operations and a collective scientific agreement on their definitions (vocabulary/repertoire/taxonomy)? 


\section{Figures}

\section{A Brain regions}

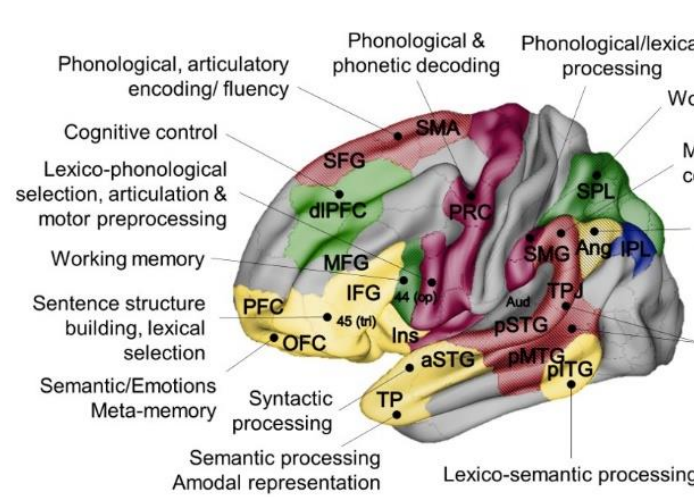

Production Comprehension/semantic Episodic/Associative Executive control

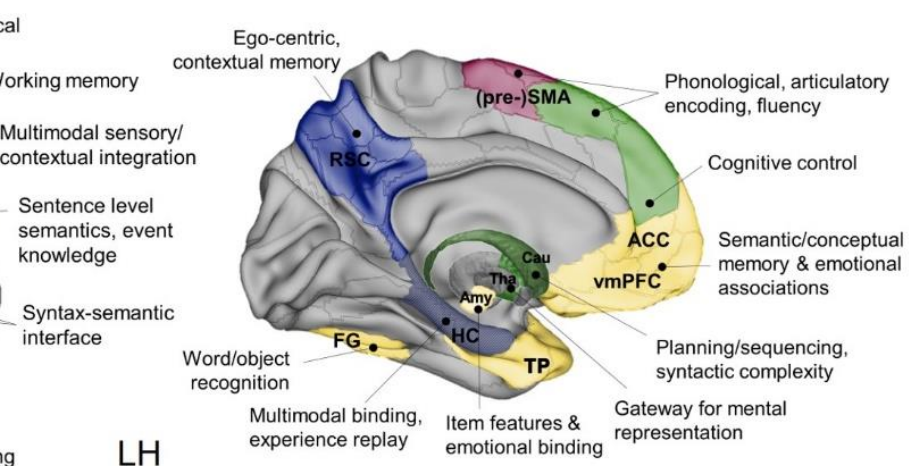

\section{B Fascicles}
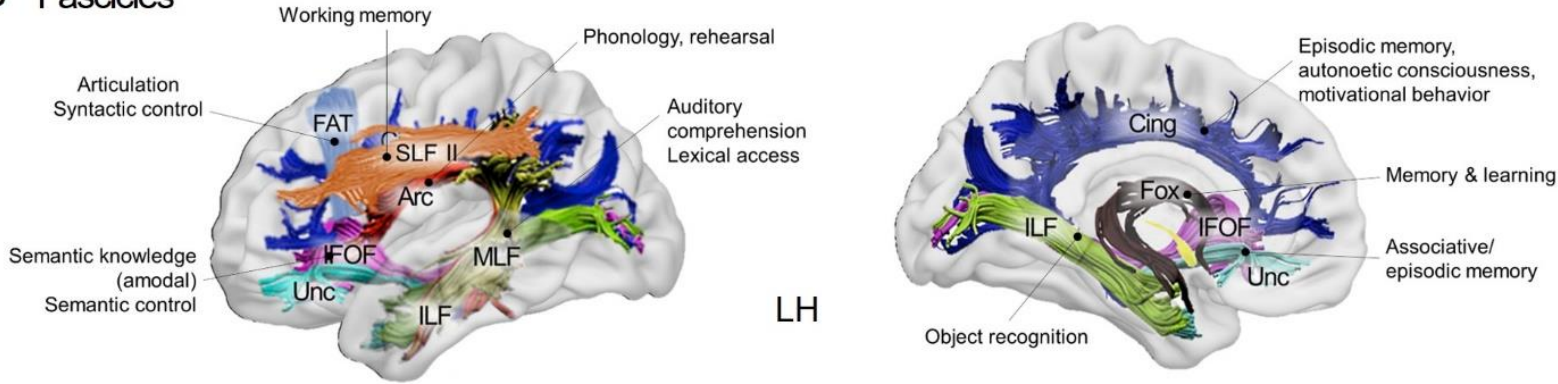

Figure 1: Main legacies of previous neurocognitive observations.

Simplified overview of the alleged functional role and specialization in language and/or memory functions of: A. Brain regions and; B. Fascicles highlighted in previous neurocognitive meta-analyses (Forkel et al., 2020; Walenski et al., 2019) or models (Duffau et al., 2014; Hagoort, 2016; Hickok \& Poeppel, 2007; Indefrey, 2011; Price, 2012), for language; (Duvernoy et al., 2013; Ranganath \& Ritchey, 2012), for memory).

\section{A Fundamental dimensions}

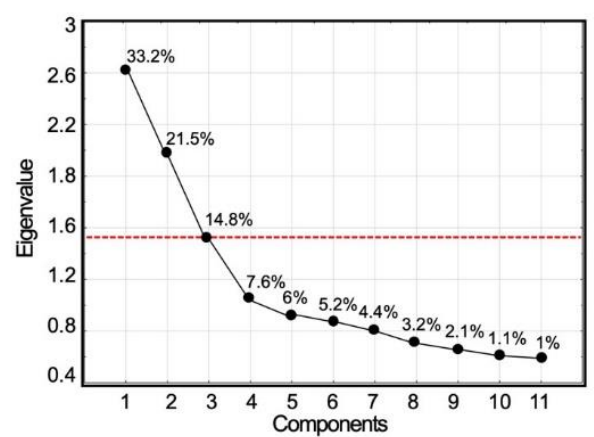

B Cognitive mapping

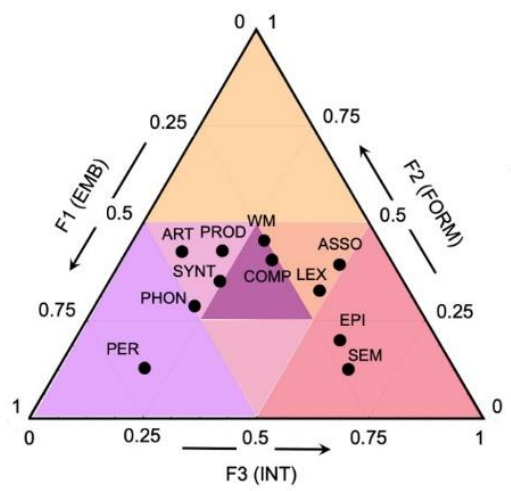

C Language-memory continuum

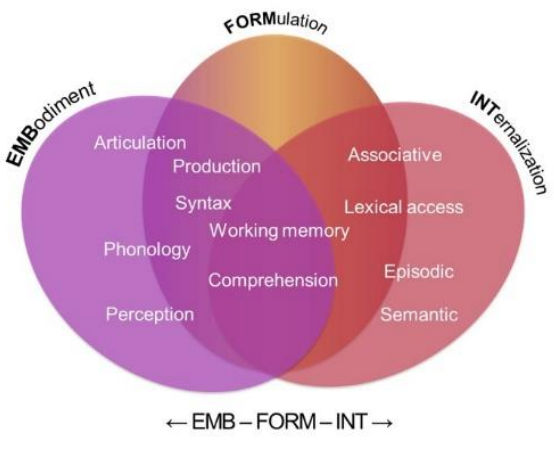

Figure 2: Neurocognitive overlap between language and memory.

Meta-analytic functional maps (positive and thresholded fMRI maps) openly provided by the Neurosynth initiative (https://neurosynth.org/; Yarkoni et al., 2011) and related to the cognitive terms associated with declarative memory and language $(\mathrm{PER}=$ speech perception; PHON = phonological, $\mathrm{SYNT}=$ syntactic; $\mathrm{ART}=$ articulatory $; \mathrm{PROD}=$ speech production; $\mathrm{WM}=$ verbal working memory; 
$\mathrm{COMP}=$ language comprehension; $\mathrm{LEX}=$ lexical; $\mathrm{ASSO}=$ associative memory; $\mathrm{EPI}=$ episodic memory; SEM = semantic memory) were analyzed using Principal Component Analysis to reveal the factorial composition of underlying language-memory behavior. A. Three principal factors emerged from the PCA (elbow, dotted red line), explaining nearly $70 \%$ of the variance (cumulative variance; scree plot). B. Projection of the cognitive constructs on the three detected dimensions. The assignment is performed on the basis of the functional representation (meta-analytic functional maps) and according to the factor loadings associated with each principal factor (ternary plot). C. The main latent dimensions in the form of a Venn diagram. The diagram is composed of three subsets that are both distinct (eigenvariance) and interrelated (common variance). The encapsulation of these modules forms the union of language-memory (LUM) behaviors, while the overlaps form the language-memory intersection $(\mathrm{L} \cap \mathrm{M})$. The three dimensions have been labeled "Embodiment (EMB) - Formulation (FORM) Internalization (INT)", according to their composition (i.e., according to the projection of the cognitive constructs; see the body of the text for a detailed description). This 3-fold reformulation is supported by observations from functional connectivity (see Figure 4), attesting to their neurobiological relevance.

\section{A Language-memory correlations}
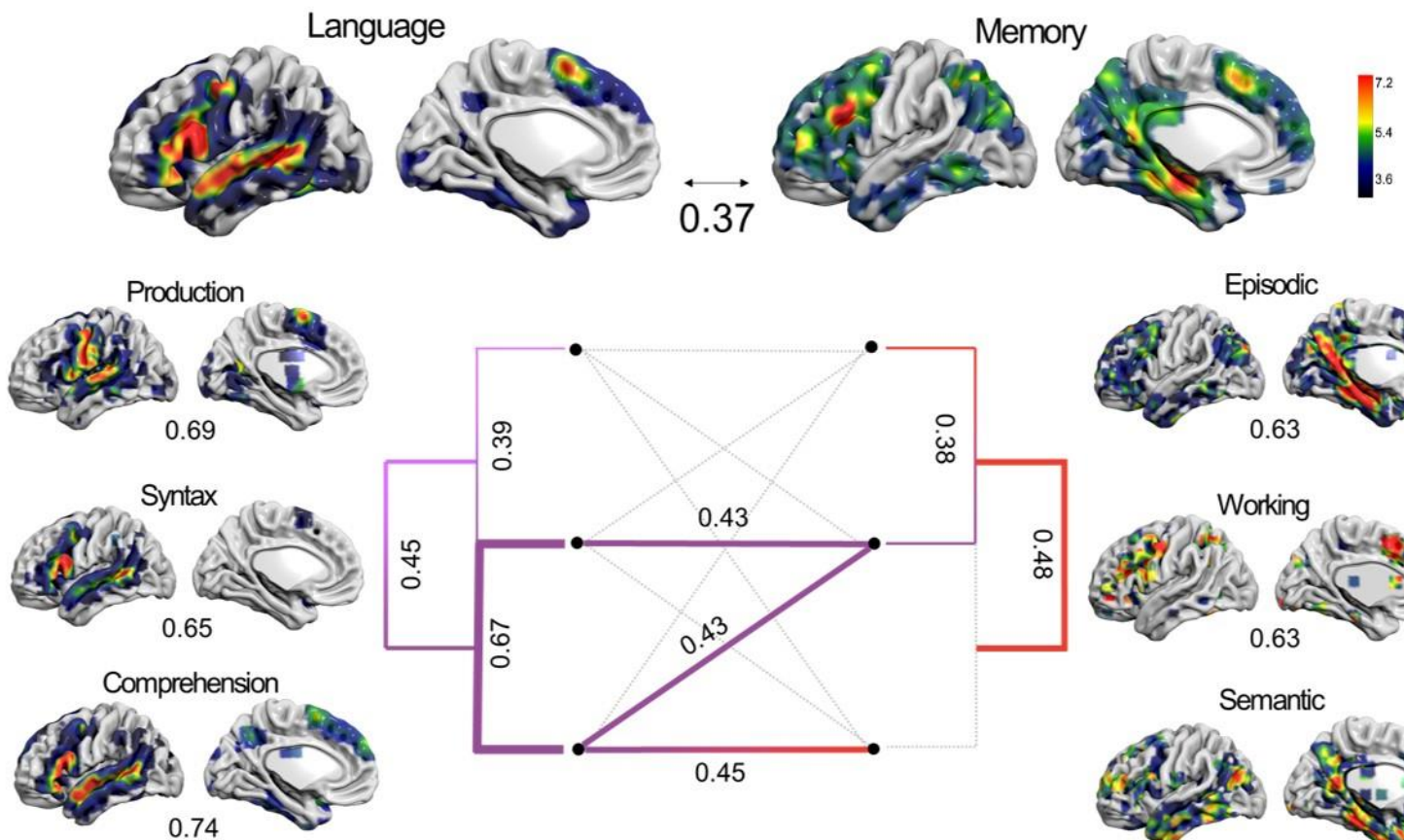

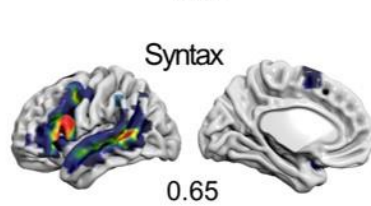

Comprehension

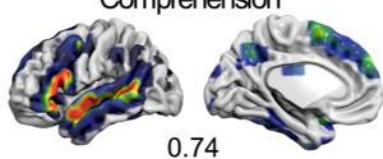

0.74

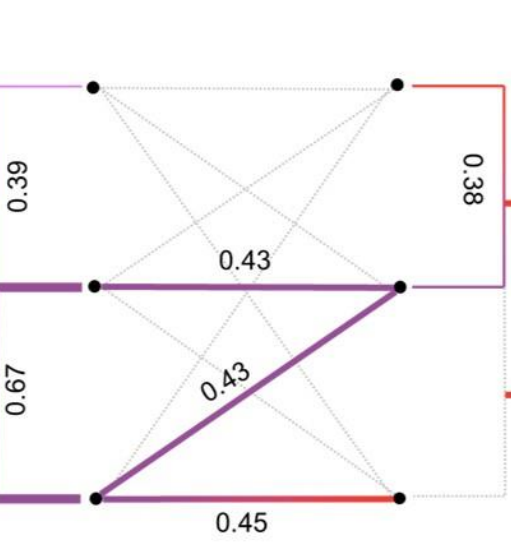

$\dot{\infty}$

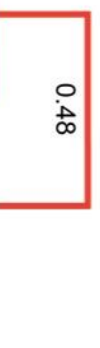

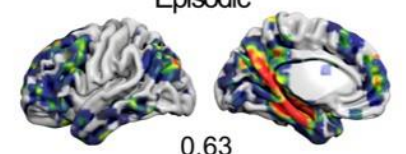

0.63

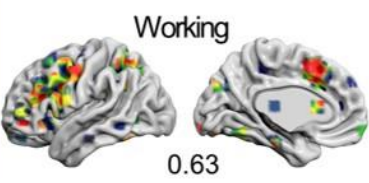

Semantic

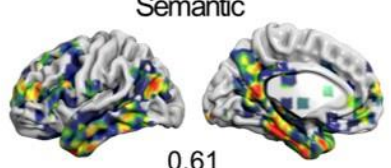

0.61

\section{B Language-memory intersection}

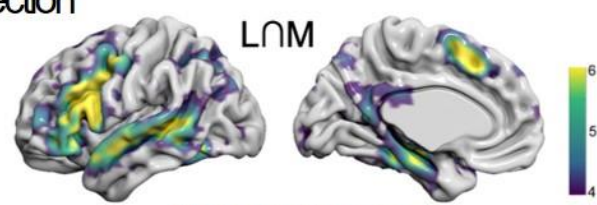

Cognitive process

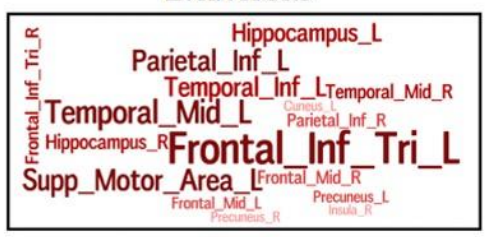

Phonologicalsyntactic Speech Semantic Words ${ }^{\text {Tasks }}$ Spoken Linguistic Comprehension Retrieval
Episodic memory Language Sentences ${ }^{D e}$ Memory verb
White matterfasides

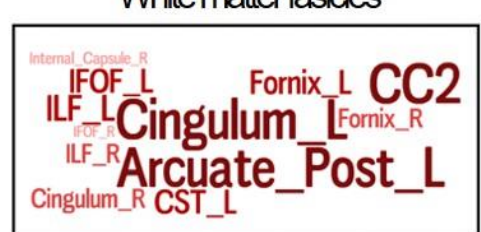

\section{Figure 3: Neurocognitive conjunction of language and memory maps.}

A. Meta-analytic language and memory functional maps openly provided by the Neurosynth initiative (https://neurosynth.org; Yarkoni et al., 2011; see also Figure 2). The strength of intra- and interfunction relationships (tanglegram) between fMRI meta-analytic maps of language (language, verbal 
production, syntax, verbal comprehension) and memory (declarative memory, episodic memory, verbal working memory, semantic memory) has been estimated using correlation coefficients. Only significant $R$-values indicating strong similarity between the maps are reported. B. Cortical regions (AAL atlas), cognitive constructs (Neurosynth decoder), and brain white matter fascicles (Natbrainlab atlas) associated with the language-memory intersection functional map ( $\mathrm{L} \cap \mathrm{M}$ mapping, computed from the functional maps included in A). The bigger the size of the words in the word clouds, the more they are linked to the functional language-memory intersection map.

\section{A Overview of the LUM model}

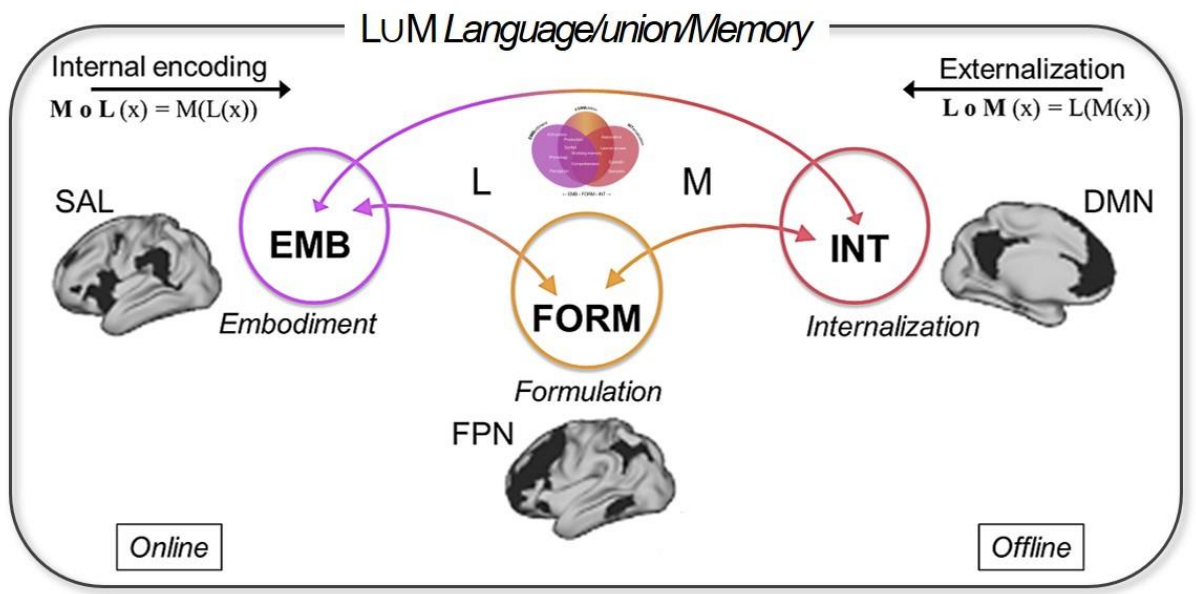

\section{B Global features}

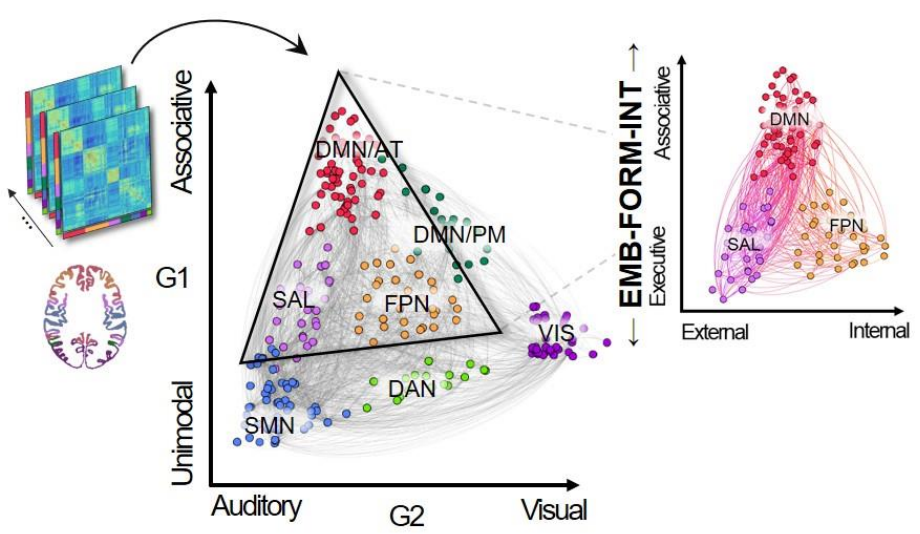

C Local features

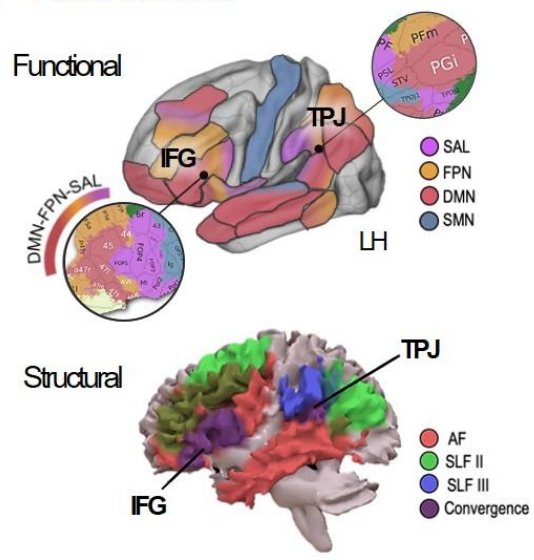

\section{Figure 4: Features and neural foundation of the LUM framework}

A. LUM model: formalization of the dynamic links spanning language production and declarative memory as an interactive oblique model (i.e., as a composite function). Latent dimensions (EMB-FORMINT) are individually associated with specific brain networks (SAL-FPN-DMN, respectively). In terms of behavior, internal encoding implied in verbal comprehension, for example, consists of encoding declarative inputs (INT) via more or less attentive listening of verbal indications (involving the EMB and FORM dimensions). Here, language "feeds" memory (MoL). Externalization, in its most accomplished form, leads to the "production" of language involving a mapping of internal verbal representations and thoughts (INT) with the corresponding ordered output forms (thus involving manipulation of FORM and EMB up to verbal evocation). Here, memory "feeds" language (LoM). To ensure dynamics between language and memory, processes engage a synergy of EMB-FORM-INT latent operations, supported by continuous interactions between networks, performed in parallel and executed in a more or less controlled manner. B. Global functional topography of the links between brain regions belonging to different networks and projected in a reduced space ( $\mathrm{n}=48$ healthy controls, at rest). This 
global topology has been observed in other recent studies (Barnett et al., 2020) and corroborates the dimensions and interactions proposed in the LUM framework (i.e., the connectivity between high-level SAL-FPN-DMN networks supporting EMB-FORM-INT linkages). C. Example of functional local SALFPN-DMN continuums (connector hubs). These functional convergence zones correspond to structural convergence zones where the terminations of traditionally described language and/or memory bundles are intertwined (Arcuate fascicle: AF; and branches II and III of the superior longitudinal fascicle: SLF II-III). Together with peripheral hubs, these connector hubs could play an important role in languagememory behavior (see description in the body text).

\section{A Pattem separation/completion}

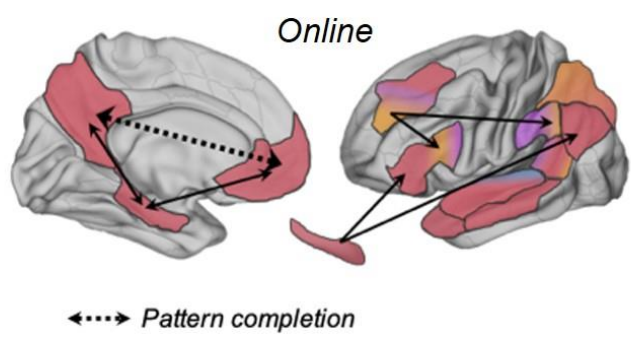

B Replay

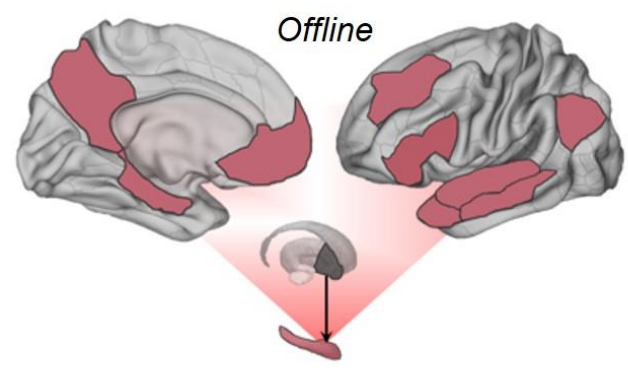

\section{Figure 5: Relational binding as one of the core neuro-mechanisms linking language and memory}

Illustration of the putative dialogues involved in the relational binding mechanism (global and local LUM dynamics). A. Pattern separation and pattern completion neurocognitive operations engage online states manifested by dialogs involving several connectors hubs. It pushes the brain to a multi-networks state between the DMN and convergence zones. Several studies have highlighted an active role of connections between the medial temporal lobe, IFG, and angular gyrus in these two processes and under the control of the dorsolateral prefrontal cortex in the integration of multiple representations (Palacio \& Cardenas, 2019 for a systematic review). Online activity recordings made at the cellular level (CA1 subfield of the rat hippocampus) show that the hippocampus engages in dynamic coordination of changes in rhythmic profiles during associative learning (Rangel et al., 2016), allowing flexible dialogues in particular with DMN regions and the convergence zones. The main difference between encoding and retrieval concerns a change in modularity, especially between the two anterior and posterior subsystems of the DMN, which are more inter-connected during memory retrieval (Cooper \& Ritchey, 2020; Geib et al., 2017). Thus, pattern completion probably engages the DMN in a more synchronized manner (dotted arrow) than pattern separation. B. The "replay" operation entails an offline state with more or less long periods of spontaneous updating of recently acquired information (Higgins et al., 2020). In terms of global LUM dialogues, consolidation is linked to an anti-phasic system associated with a strong synchronization of the DMN and limbic structures and a weaker synchronization between the DMN and other network components, such as attentional-sensory networks (Sneve et al., 2017). At the local level, suppression of thalamic activity could mediate these phase changes, reducing the transmission of external cognitive and sensory information during consolidation and allowing neocortical regions to be more receptive to input provided by peripheral hubs, mainly the hippocampus (e.g., Yang et al., 2019). 


\section{A Disruption of the global dynamics}

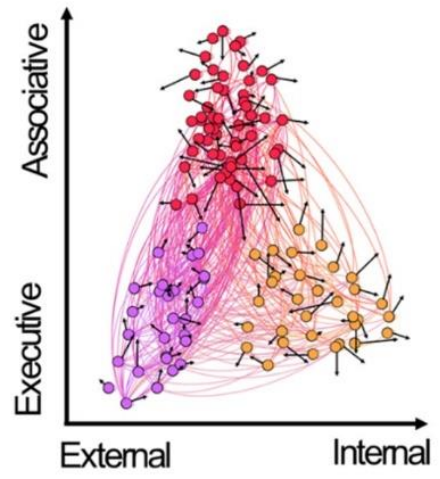

B Disruption of the local dynamics

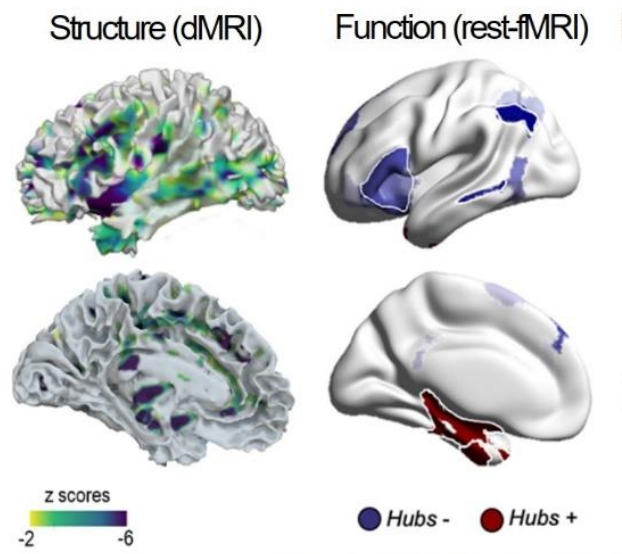

Roger et al. (2019), Hum. Brain Mapp.

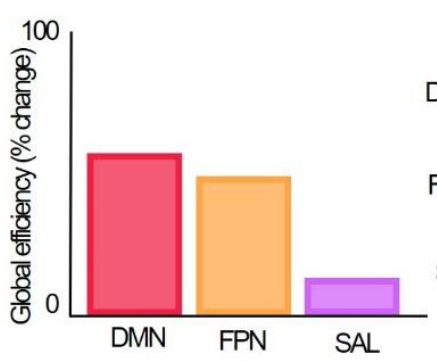

Connectivity change rate

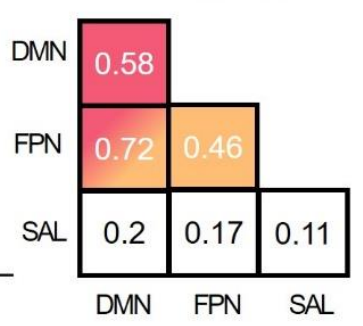

\section{Figure 6: Relevance of the LUM framework in temporal lobe epilepsy}

Temporal lobe epilepsy (TLE) is frequently symptomatic, typically accompanied by language and memory deficits (manifested in naming or verbal fluency assessment, spontaneous speech, immediate and various memory tasks (Allone et al., 2017; Bartha-Doering \& Trinka, 2014; Benke et al., 2013; Castro et al., 2013; Condret-Santi et al., 2014; Jaimes-Bautista et al., 2015; Metternich et al., 2014; Zhao et al., 2014). While there is a plurality of profiles (Reyes et al., 2020), "pure" forms of language-memory impairment are reported (Elverman et al., 2019), making TLE a relevant model for studying the LUM framework in the pathological condition. A. Disruption of global LUM dynamics in patients ( $\mathrm{n}=37$ patients compared to controls, at rest). Global changes refer to how the connectivity of brain regions belonging to a particular network is altered, thus disrupting the overall topology and synchrony. The main character is a fragmentation of the FPN and DMN networks (higher vectors of change, symbolized by the arrows), with reduced segregation between specialized networks. The global integration of information by these networks (global efficiency) is also particularly affected (boxplot). These observations are manifested by a reduction in connectivity within and between DMN-FPN networks in patients (heatmap of connectivity change rates). B. Disruption of local LUM dynamics in patients. Global LUM connectivity changes are accompanied by regional LUM disruption. At rest, patients present a significant functional disturbance of major connector and provincial hubs (namely IFG and hippocampi), essential for relational binding (LUM neuroperations; see Figure 5). These functional disturbances are indeed specifically related to poor performance in cognitive indicators requiring the associative linking of verbal information (see our previous works on TLE patients: Banjac et al., 2021; Roger et al., 2019, 2020). Moreover, the spatial pattern of functional alterations largely coincides with 
the language-memory functional intersection map (Neurosynth $\mathrm{L} \cap \mathrm{M}$, based on task-fMRI) and the alterations in structural connectivity (significant decrease in tract-weighted fractional anisotropy in patients, estimated with high-resolution diffusion MRI; Roger, 2020). Overall, TLE patients' analysis of intrinsic disturbances for both global and local dynamics leads to a relational binding syndrome hypothesis, mainly manifests by DMN-FPN de-synchronizations and essentially affecting the FORMINT LUM dimensions. These observations, in the pathological context, also confirm hypotheses about the potential interactive mechanisms involved in the relational binding operation (as proposed in Figure $5)$.

\section{Funding}

This work has been supported by the French program "AAP GENERIQUE 2017" run by the "Agence Nationale pour la Recherche" grant 'REORG' [grant number ANR-17-CE28-001501]; and by NeuroCoG IDEX UGA in the framework of the "Investissements d'avenir" program [grant number ANR-15-IDEX-02]. 